\title{
First gamma-rays from galaxy clusters. Preliminary evidence of the association of galaxy clusters with EGRET unidentified gamma-ray sources
}

\author{
S. Colafrancesco ${ }^{\star}$ \\ INAF - Osservatorio Astronomico di Roma, via Frascati 33, 00040 Monteporzio, Italy \\ Received 19 June 2001 / Accepted 28 February 2002

\begin{abstract}
The vast majority of the celestial gamma-ray sources detected so far have not yet been identified with secure counterparts at other wavelenghts. Here we report preliminary evidence for an association between galaxy clusters and unidentified gamma-ray sources of high galactic latitude $(|b|>20 \mathrm{deg})$ in the Third EGRET catalog. All of the clusters which are most probably associated with EGRET sources show evidence of strong radio emission either because they host radio galaxies/sources in their environments or because they have a radio halo or relic inhabiting their intracluster medium. The cluster radio emission suggests that the relativistic particles (electrons, protons), which are diffusing in the intracluster medium, might be also responsible for their gamma-ray emission. Beyond the spatial associations of clusters with unidentified EGRET sources, we found a correlation between the radio flux at $1.4 \mathrm{GHz}$ of the cluster's brightest source and the gamma-ray flux, $F(>100 \mathrm{MeV})$, of the associated EGRET source. For the most probable EGRET-cluster associations we also found a further correlation between the X-ray luminosity of galaxy clusters and the gamma-ray luminosity of the associated gamma-ray source under the hypothesis that the EGRET sources have the same cluster redshifts. Such correlations are consistent with the theoretical expectations and strengthen the probability of a true, physical association between galaxy clusters and gamma-ray sources.
\end{abstract}

Key words. gamma rays: observation, theory - galaxies: clusters: general - galaxies: active

\section{Introduction}

The major part of the gamma-ray sources detected with the EGRET instrument (Kanbach et al. 1988) on board the CGRO satellite have not yet been identified with secure counterparts at other wavelenghts because of the poor spatial resolution of the EGRET instrument. In fact, 170 gamma-ray sources out of the 271 found in the Third EGRET catalogue are not yet identified with firmly established counterpart (Hartman et al. 1999). Most of the unidentified gamma-ray sources are found at low galactic latitudes, $|b| \lesssim 20 \mathrm{deg}$, and are likely to belong to our Galaxy (Gehrels et al. 2000). Fifty of these sources are found at high galactic latitudes, $|b| \gtrsim 20 \mathrm{deg}$, and there are several hints that they are of extra-galactic nature (Grenier 2001). Among the identified extra-galactic gamma-ray sources observed with EGRET, most are AGNs (Hartman et al. 1999) but there is no firm evidence that the remaining unidentified EGRET sources can be associated with another population of active galaxies. In fact, most of the unidentified EGRET sources have a rather low flux variability while AGNs usually show a strong flux variability in the gamma-rays (Urry \& Padovani 1995; Ulrich et al. 1997).

Galaxy clusters are bright sources of X-rays produced through bremsstrahlung emission from a hot (with temperature $T \sim 10^{7}-10^{8} \mathrm{~K}$ ), optically thin (number density $n \sim 10^{-3} \mathrm{~cm}^{-3}$ ), highly ionized intracluster (hereafter IC) gas (mainly consisting of a population of thermal electrons and protons) in nearly hydrostatic equilibrium with the overall gravitational potential of the structure (see, e.g., Sarazin 1988 for a review). Many galaxy clusters also show the presence of non-thermal emission phenomena like extended radio halos (see, e.g., Giovannini \& Feretti 2000), likely produced by synchrotron emission of relativistic electrons either accelerated in the intracluster medium (hereafter ICM) by merging shocks or produced in the decay of dark matter annihilation products (see, e.g., Colafrancesco 2001a; Colafrancesco \& Mele 2001). Many clusters also host bright radio (or active) galaxies living in their environment. These active galaxies can inject relativistic particles into the ICM through the interaction of radio jets with the surrounding medium (Blandford 2002). The presence of relativistic particles in the ICM has been also suggested to explain the emission excesses observed in some clusters in the EUV (Lieu et al. 1999; Bowyer 2000) and in the hard X-rays (FuscoFemiano et al. 1999-2000; Rephaeli et al. 1999; Kaastra et al. 1999; Henriksen 2000). However, there is no evidence in the EGRET database for a detection of gamma-ray emission in the direction of a few selected clusters like Coma (Sreekumar et al. 1996) and Virgo.

\footnotetext{
* e-mail:cola@coma.mporzio.astro.it
} 
There are, nonetheless, several theoretical motivations to expect that galaxy clusters can indeed be extended sources of gamma-rays emitted in the decay of neutral pions, produced either in the interaction of cosmic ray protons with the ICM protons ( $p p \rightarrow X+\pi^{0} \rightarrow \gamma+\gamma$; see Colafrancesco \& Blasi 1998; Völk \& Atoyan 1999) or in the annihilation of dark matter particles $\left(\chi \chi \rightarrow X+\pi^{0} \rightarrow \gamma+\gamma\right.$; see Colafrancesco \& Mele 2001). The secondary electrons produced in the previous mechanisms (see, e.g., Blasi \& Colafrancesco 1999; Colafrancesco \& Mele 2001) can also produce additional gamma-ray emission through both bremsstrahlung and Inverse Compton Scattering (ICS) against the Cosmic Microwave Background (CMB) photons. Also primary cosmic ray electrons can produce a diffuse flux of gamma-rays due to non-thermal bremsstrahlung (see Sreekumar et al. 1996; Colafrancesco 2001b) and ICS of the CMB photons. On top of such diffuse emission, the gammaray emission emerging from individual "normal" galaxies (Berezinsky et al. 1990; Dar \& deRujula 2000) living in the cluster is also expected, as well as from "active" galaxies (Urry \& Padovani 1995) which belong to the cluster.

In this paper, we report the results of a detailed spatial and spectral analysis of the unidentified EGRET sources at high galactic latitude and the findings of preliminary evidence for a correlation between galaxy clusters and unidentified EGRET sources at $|b|>20 \mathrm{deg}$. The plan of the paper is the following. In Sect. 2 we discuss the evidence for the spatial correlation between EGRET sources at high galactic latitude and galaxy clusters in the Abell catalog (Abell et al. 1989). We also discuss the analysis of the gamma-ray flux and spectra of the EGRET sources probably associated with galaxy clusters in comparison with those associated with other EGRET sources. In Sect. 3 we analyze in details each one of the 18 EGRET sources which have possible associations with galaxy clusters. We finally derive a sample of 9 EGRET sources which are most probably associated with 12 galaxy clusters. In Sect. 4 we discuss the correlation we found between the gamma-ray flux of the EGRET source and the radio flux of the cluster radio sources for the 9 most probable EGRET-cluster associations and in Sect. 5 we discuss the evidence for a similar correlation between the gamma-ray luminosity and the X-ray luminosity of the same most probable EGRET-cluster associations. We present in Sect. 6 our conclusions and a discussion of the future expectations for the detection of gamma-ray emission from galaxy clusters in the light of the next generation space and ground-based gamma-ray experiments. We use $H_{0}=50 \mathrm{~km} \mathrm{~s}^{-1} \mathrm{Mpc}^{-1}$ and a flat $\left(\Omega_{0}=1\right)$ cosmology throughout the paper unless otherwise specified.

\section{The spatial correlation of galaxy clusters with unidentified EGRET gamma-ray sources}

Motivated by the previous arguments, we analyzed the available data for the gamma-ray sources in the Third EGRET catalog (Hartman et al. 1999) and we looked for a correlation between the position of unidentified gamma-ray sources with $|b|>20 \mathrm{deg}$ and the positions of galaxy clusters in the Abell catalogue (Abell et al. 1989). We further looked for the X-ray information about the selected clusters in the ROSAT all sky survey and pointed observations and in the BeppoSAX cluster catalogue. We also looked for radio sources associated with galaxy clusters in the NVSS radio survey, in the VLA and SUMSS surveys as well as in the available literature.

We first studied such a spatial correlation within a fixed radius ( $1 \mathrm{deg}$ ) from the center of each EGRET source and subsequently we refined our analysis considering the actual $95 \%$ confidence level position error contours of each EGRET source found in the previous step.

We found that 50 EGRET sources at high galactic latitude, $|b|>20 \mathrm{deg}$, are spatially correlated - within 1 degree from the center of the EGRET source - with the position of 70 galaxy clusters in the Abell catalogue (Abell et al. 1989). We choose a correlation radius of $1 \mathrm{deg}$ because this is the angular distance at which EGRET cannot distinguish two separate pointlike sources (Hartman et al. 1999). We performed a Monte Carlo simulation to check if such a spatial association can be understood as a simple random projection effect. Specifically, we built $10^{3}$ random distributions of galaxy clusters extracted from the Abell catalogue and taking into account their clustering properties and we cross-correlated their positions with the EGRET source positions within 1 deg radius. We find that, on average, 33 EGRET sources can be randomly associated with simulated cluster positions. Based on a Kolmogorov-Smirnov test, the probability that all of the remaining 17 EGRET unidentified sources are still randomly associated with galaxy clusters is $\$ 0.5 \%$. This indicates that the confidence level of the spatial association is about $2.96 \sigma$ (assuming a Gaussian statistics which is justified for $\gtrsim 20$ spatial associations).

For a more detailed analysis, we correlated the positions of the Abell clusters with the exact position error contours given for each EGRET source found in the Third EGRET catalogue. In this procedure we consider also the spatial extent of the galaxy clusters. We find that the coordinates of the optical centers of 52 Abell clusters fall within the contour containing the $95 \%$ confidence level error region for the positions of 39 EGRET sources. In this analysis we consider a positive correlation also for those clusters whose optical centers are close to the border of the $95 \%$ confidence level error contours of the EGRET sources and whose spatial extent is found within the 95\% confidence level EGRET position error contour. We then simulated, as before, $10^{3}$ random distributions of galaxy clusters extracted from the Abell catalogue preserving their clustering properties and we cross-correlated their positions with the EGRET source positions within their $95 \%$ confidence level contours, finding that, on average, 26 EGRET sources can be randomly associated with simulated cluster positions. Based on a Kolmogorov-Smirnov test, the probability that all of the remaining 13 EGRET unidentified sources are still randomly associated with galaxy clusters is $\lesssim 1 \%$ (or, in other words, the significance of the probable correlation between galaxy clusters and EGRET sources is at more than $2.5 \sigma$ confidence level).

Since a substantial fraction of the sky observed by EGRET has a low sensitivity (where it would be difficult to observe any faint source), the previous estimate of the significance level of the correlation can be safely considered as a lower limit of the true one. In fact, the cluster - EGRET source correlation we found here is suffering from a lack of 
other possible EGRET-cluster associations coming from those gamma-ray sources which are not detected in the low-exposure region of the EGRET sky. Assuming that the number of additional EGRET sources detectable with a uniform sky coverage, $N_{\mathrm{x}} \propto A_{\text {low-exp. }}$ (where $A_{\text {low-exp. }}$ is the area of the gamma-ray sky with low-exposure), is correlated with galaxy clusters in the same ratio of our previous estimates, and assuming that the fraction of random correlation is again similar to what previously estimated (i.e. $\sim 2 / 3$ of the correlations are random and $\sim 1 / 3$ are probable), the statistical confidence level of the correlation found after correcting for the non-uniform exposure of EGRET increases with increasing value of $N_{\mathrm{x}}$ and scales like $\sim \sqrt{N_{\mathrm{x}}} \propto \sqrt{A_{\text {low-exp. }}}$, for high values of $N_{\mathrm{x}}$. So, in conclusion, we believe that the previous estimate of the statistical significance of the cluster-EGRET source correlation given above can be reliably considered as a lower limit to the actual significance level of the spatial correlation between galaxy clusters and unidentified EGRET sources.

To select out of the full list previously found the more probable associations of galaxy clusters with the unidentified EGRET sources, we superposed the optical cluster positions and their X-ray images onto the maps containing the probability distribution for the spatial position of the 50 EGRET sources found in our spatial correlation analysis.

We found that 18 of the original 50 EGRET sources associated with galaxy clusters have also an AGN (with confirmed identification) whose position falls within the $95 \%$ confidence level position error contours of the gamma-ray source. We also found that a Gamma Ray Burst is found in association with the EGRET source 3EG J2255-5012 and the clusters Abell 1073S and Abell 1074S. Also a SN remnant is found in the field of the source 3EG J1235+0233 associated to the cluster Abell 1564. We then excluded these 20 EGRET sources and the associated 30 clusters from the list of probable physical associations.

We also excluded 12 EGRET sources with a possible, but not confirmed, AGN contamination in the Third EGRET cata$\log$ (see Hartman et al. 1999). Note that also this procedure is very conservative since there are 4 cases out of the 12 listed in which the possible AGN source is found beyond the $95 \%$ confidence level position error contours of the associated EGRET sources, while the galaxy clusters spatially associated with the EGRET sources fall within their $95 \%$ confidence level position error contours.

Finally, we conclude that, in our conservative analysis, 24 galaxy clusters are associated to 18 unidentified EGRET sources with $|b|>20 \mathrm{deg}$ for which there is no firmly established counterpart at other wavelengths, neither extragalactic (AGN or "active" galaxy) or galactic (Supernova remnant, pulsar, neutron star). All of these galaxy clusters have their optical and X-ray centers falling within the $95 \%$ confidence level position error contours of the EGRET sources. We show in Table 1 the list of the 18 EGRET sources and the 24 clusters which are spatially correlated within the $95 \%$ confidence level position error contours of each EGRET source. This is the initial sample of likely associations between galaxy clusters and EGRET gamma-ray sources on which we performed a more detailed analysis, as discussed in the following.
According to our selection procedure, the significance level of such a spatial association is $\approx 2.55 \sigma$ which corresponds to a probability $\lesssim 1 \%$ for the null hypothesis that the two source populations are randomly associated. However, the point is still to determine how many of these spatial associations are due to random projection effects and which are the most probable physical associations. A rough estimate of the probability to have still random associations in the sample here selected (see Table 1) and to be not contaminated by either extra-galactic (AGN, GRB) or galactic (SNR, pulsars, ...) gamma-ray sources, yields that about $2 / 3$ of the 18 selected EGRET sources are still random associations. This rough estimate would yield 6 most probable cluster-EGRET source associations with a confidence level of $1.73 \sigma$. Note, however, that this is again a lower limit to the true statistical confidence of the correlation since the effect of the non-uniform EGRET sky coverage has to be taken into account and would tend to increase the statistical significance level of the most probable association. If we correct for the number of correlations expected in the fraction of the EGRET sky ( 30\% of the full sky) which has a flux limit below $F(>100 \mathrm{MeV}) \leq 6 \times 10^{-8} \mathrm{~cm}^{-2} \mathrm{~s}^{-1}$, we obtain that the expected confidence level of the most probable associations increases from $1.73 \sigma$ to $2.12 \sigma$.

\subsection{Flux and spectral analysis}

In addition to the spatial information contained in the Third EGRET catalog and in the Abell cluster survey, we can use more physical criteria to determine the number of spurious correlations in our selected sample of Table 1. Specifically, we first analyze the flux level, the flux variability and the spectral indices of the 18 EGRET sources in Table 1 compared to the same quantities of other gamma-ray sources more definitely identified in the Third EGRET catalogue (mainly AGN and Pulsars). Then we run Monte Carlo simulations of flux level and variability for the probable EGRET-cluster associations to determine the fraction of random correlations expected in our selected sample.

Figure 1 shows the flux variation in the viewing periods (hereafter VP) over which the EGRET sources reported in Table 1 have been detected. We notice that the flux variability for the probable cluster-EGRET source associations listed in Table 1 is, on average, $\$ 20 \%$ and only in a few cases (3EG J1825-7926, 3EG J1212+2304, 3EG J0616-3310, $3 E G \mathrm{~J} 2248+1745$ ) it can be considered $\gtrsim 30 \%$ in some specific VP (see Fig. 1). The correspondingly associated clusters (see Table 1) are poorly studied, do not have X-ray information and do not have any identified bright radio galaxy or radio halo/relic emission. Hence, we also consider these cases as suspiciously due to projection effects. Beyond the positive detections with high statistical significance $(T S)^{1 / 2} \gtrsim 4$ (see Hartman et al. 1999 for a definition of the quantity $(T S)^{1 / 2}$ ) of the EGRET sources reported in Fig. 1, the Third EGRET catalog provides also upper limits on their fluxes in other independent VPs. Such upper limits have $(T S)^{1 / 2}<2$ (i.e., a low statistical significance) and we verified that most of them are consistent with the positive detections of the EGRET sources we show 
Table 1. List of probable cluster - EGRET source association. Shown are the coordinates (J2000) of the EGRET unidentified sources (Cols. 2 and 3) together with those of the associated galaxy clusters (Cols. 5 and 6). The cluster optical redshifts (Col. 7), their richnesses $R$ (Col. 8) and optical radii $r_{\text {opt }}$ (Col. 9) are extracted from the NED archive, unless otherwise specified (the redshift references are: ${ }^{a}$ Andernach 2002, priv. comm.; ${ }^{k}$ Kowalski et al. 1984). The most probable associations are marked with an asterisk (see text for details).

\begin{tabular}{|c|c|c|c|c|c|c|c|c|c|}
\hline EGRET source & RA & Dec & Cluster & RA & Dec & $z$ & $R$ & $r_{\mathrm{opt}}$ & Notes \\
\hline \multirow[t]{2}{*}{ *3EG J2219-7941 } & 222000.0 & -794124.00 & Abell 1014S & 222410 & -80104 & 0.048 & 0 & - & SUMSS \\
\hline & & & Abell 1024S & 222732 & -78454 & $0.105^{a}$ & 0 & - & SUMSS \\
\hline 3EG J1825-7926 & 182502.4 & -792624.00 & Abell 3631 & 183408 & -78474 & $0.085^{a}$ & 0 & - & SUMSS \\
\hline 3EG J0348-5708 & 034828.8 & -570824.00 & Abell 3164 & 034549 & -57024 & 0.057 & 0 & $4.5^{\prime}$ & SUMSS \\
\hline \multirow[t]{2}{*}{ 3EG J0159-3603 } & 015921.6 & -360336.00 & Abell 2963 & 020045 & -35593 & $0.113^{a}$ & 0 & - & NVSS \\
\hline & & & Abell 219S & 020203 & -35483 & $0.128^{a}$ & 1 & $15^{\prime}$ & - \\
\hline \multirow[t]{3}{*}{ 3EG J0616-3310 } & 061636.0 & -331012.00 & Abell 577S & 061518 & -34070 & $0.102^{a}$ & 2 & $7^{\prime}$ & NVSS \\
\hline & & & Abell 575S & 061325 & -33405 & $0.098^{a}$ & 0 & - & NVSS \\
\hline & & & Abell 573S & 061202 & -32574 & $0.078^{a}$ & 0 & - & NVSS \\
\hline 3EG J2034-3110 & 203455.2 & $\begin{array}{lll}-31 & 10 & 48.00\end{array}$ & Abell 886S & 203711 & -31383 & $0.095^{a}$ & 0 & - & - \\
\hline \multirow{2}{*}{ 3EG J1234-1318 } & 123402.4 & -131836.00 & Abell 1558 & 123359 & -13343 & $0.116^{a}$ & 0 & $14^{\prime}$ & NVSS \\
\hline & & & Abell 1555 & 123159 & -13233 & $0.127^{a}$ & 1 & $14^{\prime}$ & NVSS \\
\hline 3EG J0038-0949 & 003857.6 & -094912.00 & Abell 85 & 004137 & -09203 & 0.056 & 1 & $30^{\prime}$ & RH, RG, NVSS \\
\hline 3EG J1310-0517 & 131024.0 & -051800.00 & Abell 1688 & 131129 & -04405 & $0.190^{k}$ & 0 & - & NVSS \\
\hline 3EG J0253-0345 & 025357.6 & -034536.00 & Abell 388 & 025136 & -03454 & 0.134 & 2 & $10^{\prime}$ & NVSS \\
\hline 3EG J0439+1105 & 043914.4 & 110524.00 & Abell 497 & 043651 & 10380 & $0.140^{k}$ & 0 & $17.5^{\prime}$ & NVSS \\
\hline $3 E G$ J0215+1123 & 021600.0 & 112248.00 & Abell 331 & 021535 & 11215 & $0.186^{k}$ & 1 & $9^{\prime}$ & NVSS \\
\hline $3 E G$ J2248+1745 & 224857.6 & 174612.00 & Abell 2486 & 224845 & 17095 & $0.143^{k}$ & 0 & $18^{\prime}$ & NVSS \\
\hline $3 E G$ J1212+2304 & 121236.0 & 230448.00 & Abell 1494 & 121314 & 23561 & $0.159^{k}$ & 1 & $15^{\prime}$ & NVSS \\
\hline $3 E G$ J1347+2932 & 134712.0 & 293224.00 & Abell 1781 & 134428 & 29505 & 0.062 & 0 & $16^{\prime}$ & RG, NVSS \\
\hline \multirow[t]{2}{*}{ 3EG J1424+3734 } & 142452.8 & 373448.00 & Abell 1902 & 142146 & 37172 & 0.160 & 2 & $15^{\prime}$ & RG, NVSS \\
\hline & & & Abell 1914 & 142602 & 37493 & 0.171 & 2 & $13^{\prime}$ & $\mathrm{RH}, \mathrm{RG}, \mathrm{NVSS}$ \\
\hline 3EG J1337+5029 & 133731.2 & 502848.00 & Abell 1758 & 133232 & 50303 & 0.279 & 3 & $11^{\prime}$ & RH, RG, NVSS \\
\hline 3EG J1447-3936 & 1414748.0 & -393636.0 & Abell 774S & 144923 & -40206 & $0.062^{a}$ & 0 & - & - \\
\hline
\end{tabular}

Notes: RG: identified radio galaxies in the cluster; NVSS, SUMSS: radio sources found within the Abell radius, $\frac{1.7}{Z}$ arcmin, of the cluster; RH: radio halo or relic belonging to the cluster. The specific ID names of the identified radio galaxies and NVSS radio sources associated to the clusters are given in Sect. 3 of the text.
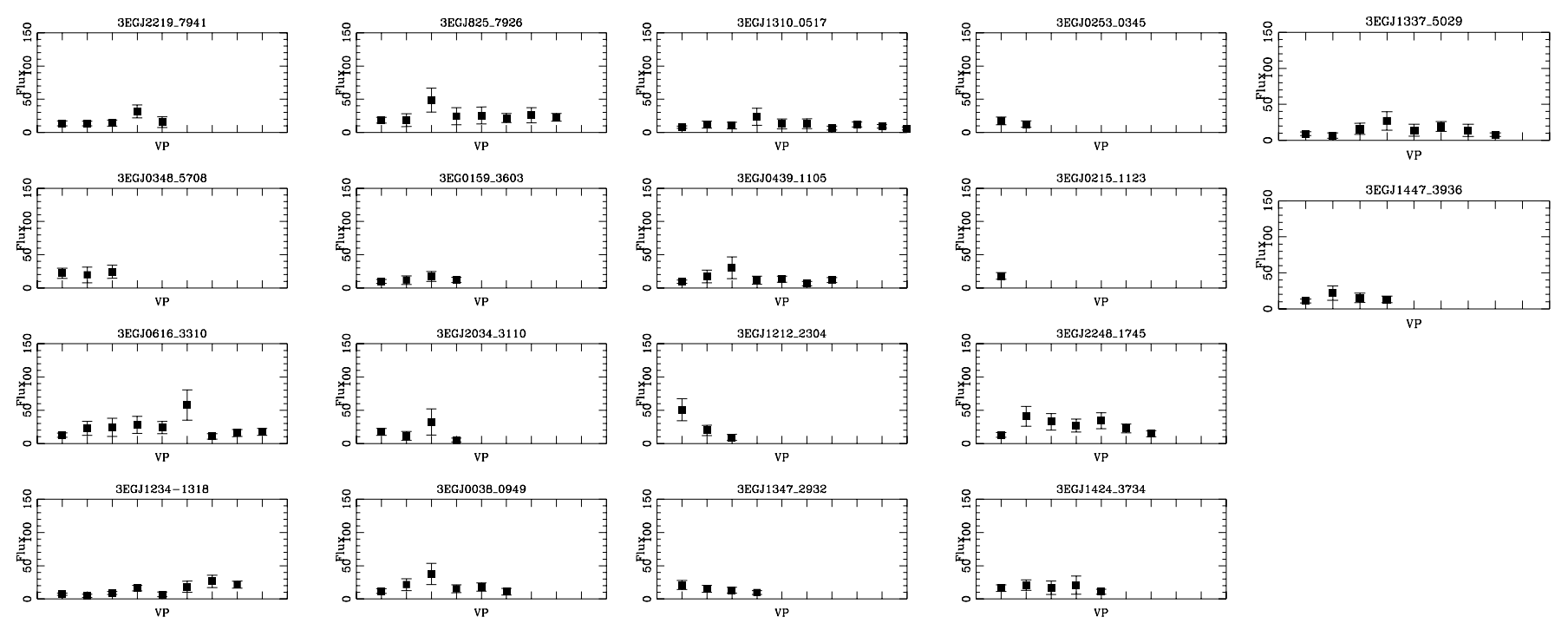

Fig. 1. We show the gamma-ray flux of the EGRET sources in Table 1 as detected in the different viewing periods (VP) of the source detection. Data are from Hartman et al. (1999). The flux detected in the different VPs are reported here in the sequential order given in the Third EGRET catalog, being the correct observing time sequence irrelevant for our purposes. The fluxes of the EGRET sources are in units of $10^{-8} \mathrm{~cm}^{-2} \mathrm{~s}^{-1}$. 
in Fig. 1. In some cases, however, (see, e.g., 3EGJ0348-5708, 3EGJ1234-1318, 3EGJ0253-0345, 3EGJ0215+1123) there are upper limits which are well below the flux level found in other independent VP detections of the sources. Nonetheless, we noticed that these "quite low" upper limits all have a very low statistical confidence level, $(T S)^{1 / 2} \sim 0$, and are hence extremely unreliable. Thus, due to their quite low statistical significance, the upper limits of the EGRET sources listed in Table 1 and shown in Fig. 1 do not strongly affect our conclusions on their overall flux variability. A few other sources with independent flux upper limits below the definite detections (see, e.g., 3EGJ0616-3310, 3EGJ2034-3110, 3EGJ1212+2304) show also a level of flux variability which does not justify to consider them as stationary sources. For the sake of completeness, we will discuss in Sect. 3 below the detailed analysis of each specific EGRET source listed in Table 1.

For comparison, we show in Fig. 2 the flux variation of the EGRET sources which are correlated with galaxy clusters and moreover contain also a confirmed AGN in the field. In these last cases, the flux of the EGRET sources not only show stronger and statistically significative variations, but also have a much higher value of their average gamma-ray flux.

In Fig. 3 we compare the spectral index, $\gamma$, of the EGRET sources which are probably associated with galaxy clusters with those of the EGRET sources which are spatially correlated with galaxy clusters and moreover contain an AGN in the field. The EGRET sources correlated with clusters are not found to be brighter than $F(>100 \mathrm{MeV}) \sim 2 \times 10^{-7}$ counts $\mathrm{cm}^{-2} \mathrm{~s}^{-1}$ and show spectral indices in a large range $\sim 2 \times 3.5$. With a remarkable difference, the EGRET sources identified with known AGNs span over a much higher gamma-ray flux range and have a much smaller range of spectral index values $(\gamma \sim 2-$ $2.5)$ especially at very bright flux levels $F(>100 \mathrm{MeV})>$ $5 \times 10^{-7}$ counts $\mathrm{cm}^{-2} \mathrm{~s}^{-1}$. Pulsars also show very flat spectral indices $\gamma \lesssim 2$ and very high gamma-ray flux which cannot be compared with those of the EGRET sources associated with clusters.

The gamma-ray spectral indices for the probable associations listed in Table 1 have values which are consistent with those expected from the viable mechanisms for gamma-ray emission in clusters. Theoretical models for cluster gamma-ray emission predict in fact slopes in the range $\gamma \sim 1.8-3.2$, going from annihilation of dark matter neutralinos (Colafrancesco \& Mele 2001) to non-thermal electron bremsstrahlung (Colafrancesco 2001a,b; Blasi 2000). Only the sources 3EG J2034-3110 (associated to Abell 886S) and 3EG J1424+3734 (associated to Abell 1902 and Abell 1914) have spectral indices $\gtrsim 3$, even though with large uncertainties. However, while the first source, 3EG J2034-3110, shows also some level of flux variability (see Fig. 1) and could then be contaminated by AGN-like sources, the gamma-ray source 3 EG J1424+3734 has a very low flux variability $(\sim 15 \%)$ and is likely to be a probable association whose gamma-ray emission could be dominated by non-thermal electron bremsstrahlung, which shows typically a steep spectrum consistent with that of the parent cosmic-ray electrons (see, e.g., Longair 1993).

We finally run Monte Carlo simulations of the flux variability level of the 18 EGRET sources of Table 1. For a uniform random distribution of their fractional flux change, $\Delta F / F$, we expect 4 EGRET sources with $\Delta F / F \lesssim 0.2$, while the remaining 14 EGRET sources possibly associated with galaxy clusters should have $0.2 \lesssim \Delta F / F \lesssim 1$. The actual data reported in Fig. 1 show that there are about 11 EGRET sources with $\Delta F / F \leq 0.2$ and only 7 sources with $0.2 \lesssim \Delta F / F \lesssim 1$. This indicates that the low flux variability shown by the EGRET sources found in association with clusters cannot be recovered by a simple random distribution at more than the $5 \sigma$ confidence level.

Based on these results we expect that about 10 EGRET sources out of the 18 listed in Table 1 are probable EGRETcluster associations having $\Delta F / F \lesssim 0.2, F(>100 \mathrm{MeV})<$ $(1-2) \times 10^{-7} \mathrm{~cm}^{-2} \mathrm{~s}^{-1}$ and $\gamma \sim 2-3.2$. However, only a detailed analysis of the spatial and spectral features of each EGRET source as well as a detailed analysis of their cluster counterparts can reveal the nature of the more probable physical association. We will present in the next section the detailed analysis of each one of the specific EGRET sources listed in Table 1 and of their possible astrophysical counterparts.

\section{Analysis of the specific sources}

In this section we analyze in details the more probable associations of galaxy clusters with the unidentified EGRET sources which are listed in Table 1: all of these galaxy clusters are found within the 95\% confidence level position error contours of the associated EGRET source. As for the flux of each source, we report only the first entry given in the Third EGRET catalogue (Hartman et al. 1999) providing also the viewing period (VP) of the source detection and the significance level, $(T S)^{1 / 2}$, of the detection. The reader may refer to Hartman et al. (1999) for the full list of information about the EGRET source under consideration.

\subsection{EG J2219-7941}

This EGRET source has been detected with a $(T S)^{1 / 2}=4.4$ in the $\mathrm{VP}=\mathrm{P} 1234$. It has a flux $F_{\mathrm{P} 1234}(>100 \mathrm{MeV})=(13.5 \pm 3.6) \times$ $10^{-8} \mathrm{~cm}^{-2} \mathrm{~s}^{-1}$ with a power-law spectral index $\gamma=2.50 \pm 0.29$. Its flux does not change significantly over all the VPs with the exception of the VP=10.0 in which a flux increase by a factor $\sim 2$ has been recorded; however, this flux is consistent with the flux detected in the other periods at the $2 \sigma$ confidence level, so that it can be considered a stationary gamma-ray source (see Fig. 1). There is no identified gamma-ray source counterpart for this EGRET source and, thus, it is a good candidate for the galaxy cluster association. Two Abell clusters fall within the 95\% confidence level position error contours of this source: Abell 1014S and Abell 1024S. The EGRET source map is very broad with an effective radius of the $95 \%$ confidence level position error circle of $\theta_{95}=0.63 \mathrm{deg}$. The elongation of the EGRET source probability map is aligned with the position of the two clusters, suggesting a possible contribution to the gamma-ray emission from both clusters (see Fig. 4).

There is no definite X-ray information on these two galaxy clusters available in the literature. However, we found that both these clusters have some radio information. Given the low declination of the sources, they are not covered by the NVSS. 

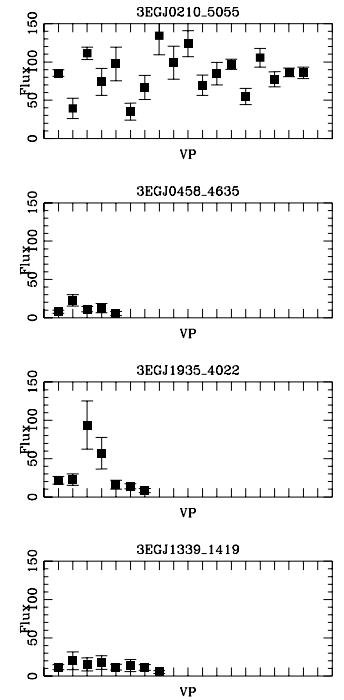
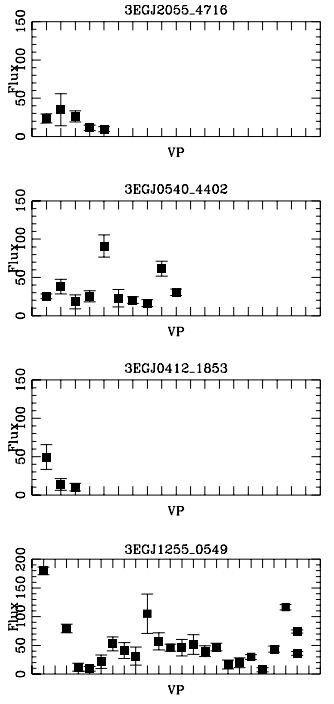
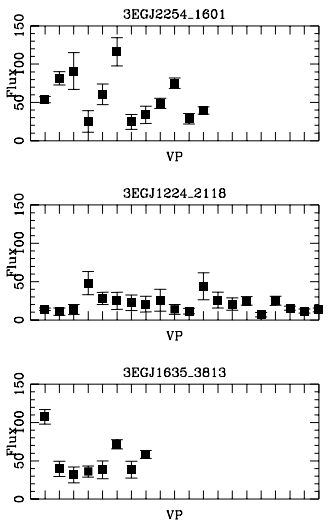

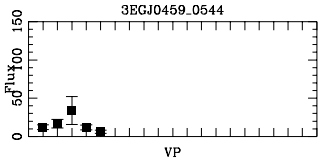

3EGJ1605_1553
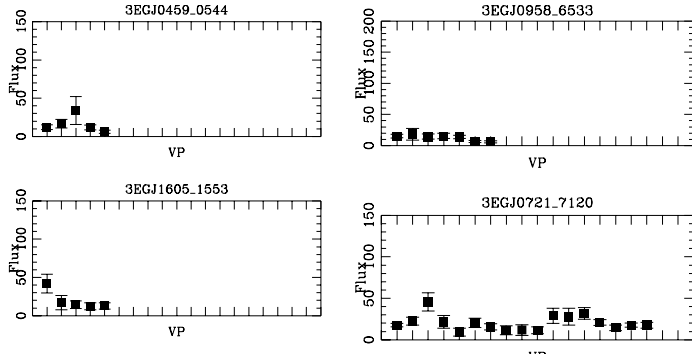

Fig. 2. The variation of the gamma-ray flux of the EGRET sources which are correlated with galaxy clusters and which have also an identified AGN in the field. Data are from Hartman et al. (1999). As in Fig. 1, the flux detected in the different VPs are reported in the sequential order given in the Third EGRET catalog, being the correct observing time sequence irrelevant for our purposes. The fluxes of the EGRET sources are in units of $10^{-8} \mathrm{~cm}^{-2} \mathrm{~s}^{-1}$.
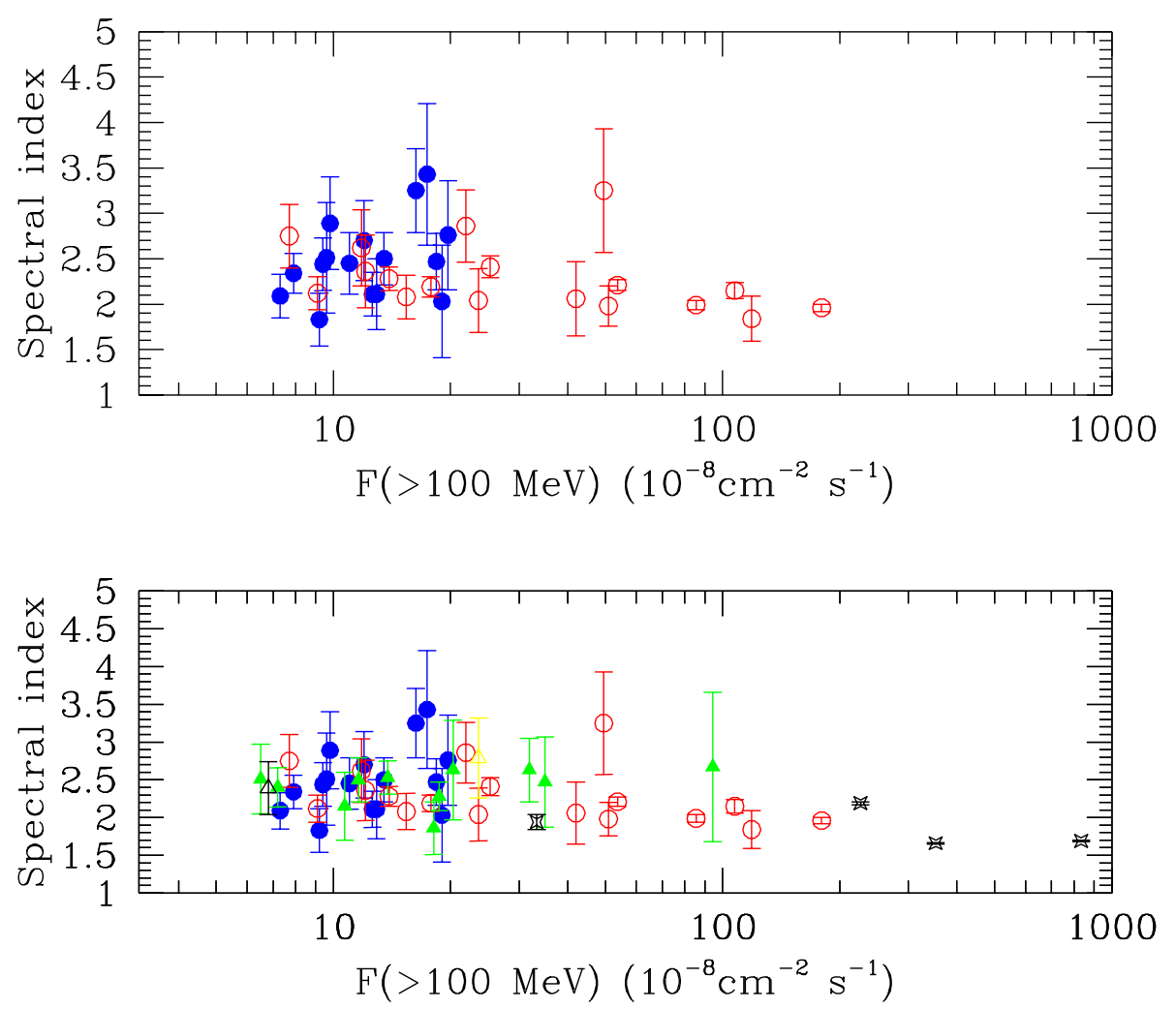

Fig. 3. The gamma-ray spectral index $\gamma$ is plotted against the gamma-ray flux $F(>100 \mathrm{MeV})$ of the EGRET sources found to be spatially correlated with galaxy clusters. In the top panel we compare the spectral indices for the EGRET sources which are more probably associated with clusters (filled circles) with the EGRET sources again correlated with clusters and in which an identified AGN is also found (open circles). In the lower panel the data from the EGRET sources contaminated by possible AGNs (gray triangles), GRB (open, light-gray triangle), SNR (open black triangle) and Pulsars (stars) are added to the previous data sets. Data are from Hartman et al. (1999).

So, we looked for radio sources associated with the cluster in the SUMMS survey (Bock et al. 1999). We found that Abell 1014S at $z=0.048$ (Abell et al. 1989) has several bright SUMSS radio sources located within its Abell radius,
$\frac{1.7}{Z}$ arcmin. A preliminary analysis of the RASS field also yielded a flux $F_{0.5-2.5 \mathrm{keV}} \lesssim 4.1 \times 10^{-12} \mathrm{erg} \mathrm{cm}^{-2} \mathrm{~s}^{-1}$.

The cluster Abell 1024S has an estimated redshift of $z=$ 0.1053 (taken from a 2002 version of Andernach \& Tago 1998 

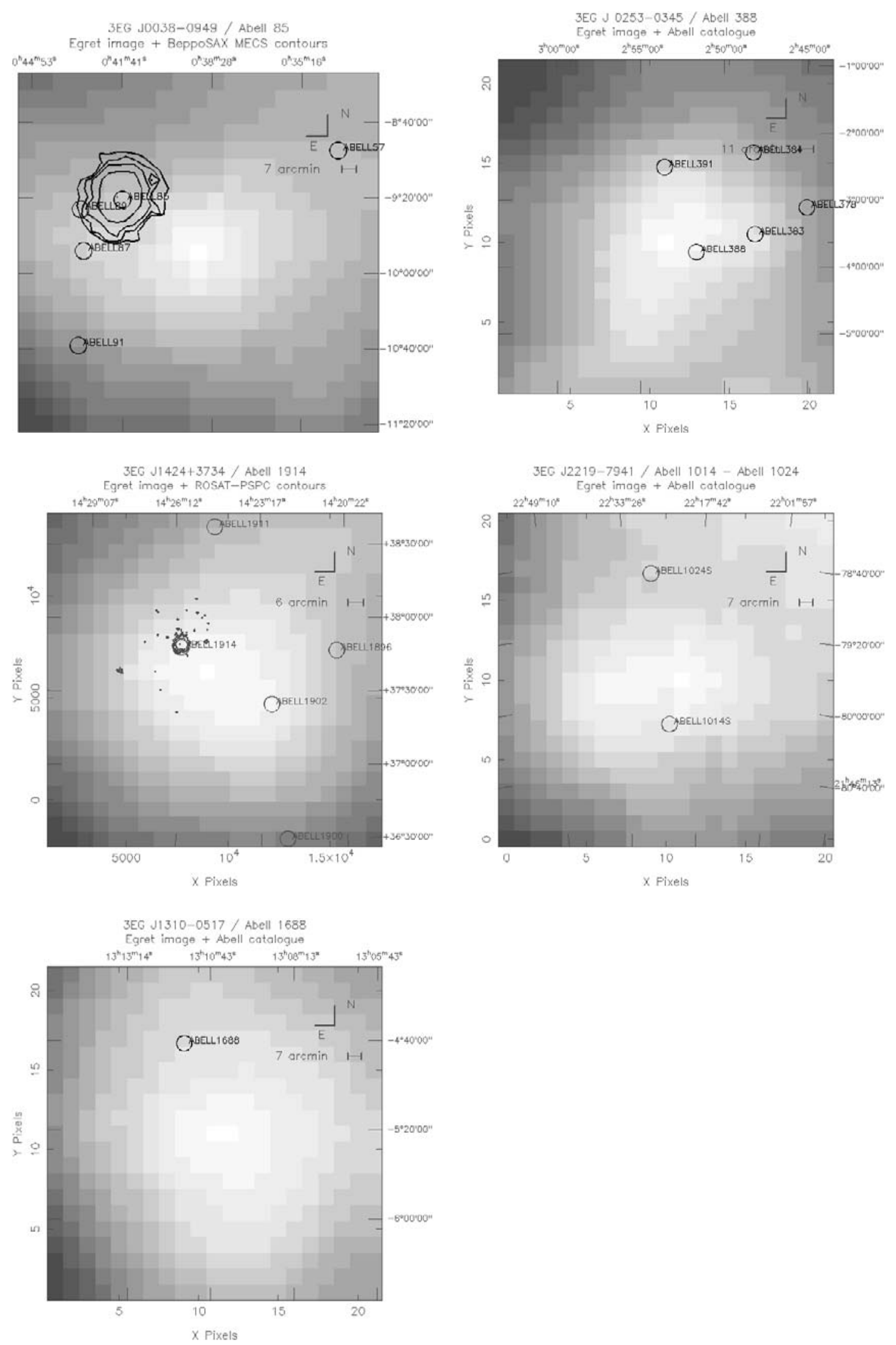

Fig. 4. We show here the maps of the galaxy clusters which are more probable candidates for the associations with unidentified EGRET sources: 3EGJ0038-0949 associated with Abell 85 (upper left), 3EGJ0253-0345 associated with Abell 388 (upper right), 3EGJ1424+3734 associated with Abell 1914 and Abell 1902 (mid left), 3EGJ2218-7941 associated with Abell 1024S and Abell 1014S (mid right) and 3EGJ1310-0517 associated with Abell 1688 (bottom left). The cluster positions and X-ray brightness contours (when available) are superposed to the maps of the EGRET sources. The intensity scale of the EGRET maps goes from black (minimum) to white (maximum). 
compilation of Abell clusters redshifts, H. Andernach 2002, priv. comm.) and we found two bright SUMSS radio sources within its Abell radius.

A preliminary analysis of all these radio sources yields a total flux evaluated at $1.4 \mathrm{GHZ}$ of $S_{1.4} \approx 270 \mathrm{mJy}$. A more refined analysis of the radio and X-ray sources in the field of this EGRET source will be presented in a future paper Colafrancesco \& Andernach (2002, in preparation).

Based on the previous evidence, we consider now that this is a probable association between galaxy clusters and an EGRET gamma-ray source.

\section{2. $3 E G ~ J 1825-7926$}

This EGRET source has been detected with a $(T S)^{1 / 2}=4.9$ in the $\mathrm{VP}=\mathrm{P} 1234$. It has a flux $F_{\mathrm{P} 1234}(>100 \mathrm{MeV})=(18.4 \pm$ 4.5) $\times 10^{-8} \mathrm{~cm}^{-2} \mathrm{~s}^{-1}$ with a power-law spectral index $\gamma=$ $2.47 \pm 0.31$. Its flux does not change significantly over all the VPs with the exception of the VP=38.0 in which a flux increase by a factor $\sim 2$ has been recorded; however, this flux is consistent with the flux detected in the other periods at less than the $2 \sigma$ confidence level (see Fig. 1). The quite low upper limit of $<13.5 \times 10^{-8} \mathrm{~cm}^{-2} \mathrm{~s}^{-1}$ found in the $\mathrm{VP}=402$.+ has $(T S)^{1 / 2}=0$ and thus is not statistically significant. The Abell cluster Abell 3631 falls at the border of the 95\% confidence level position error contours of the EGRET source. The position error map of this EGRET source is quite broad with $\theta_{95}=0.78 \mathrm{deg}$ and it is elongated in the south-west north-east direction. There is no other known gamma-ray source counterpart for this EGRET source. However, there is poor information available on the cluster Abell 3631. In particular, there is no NVSS radio source found in the field of this cluster and no $\mathrm{X}$-ray information. There is one bright radio source in the field of 3EGJ1825-7926 found in the SUMSS survey, but it is $\sim 20$ arcmin away from the optical center of Abell 3631 .

Due to the previous evidence, there is no strong hint indicating the possible association of this cluster with the EGRET source 3EG J1825-7926 and so we consider this case as likely due to projection effects.

\subsection{EG J0348-5708}

This EGRET source has been detected with a $(T S)^{1 / 2}=4.1$ in the $\mathrm{VP}=\mathrm{P} 2$. It has a flux $F_{\mathrm{P} 2}(>100 \mathrm{MeV})=(22.1 \pm 7.6) \times$ $10^{-8} \mathrm{~cm}^{-2} \mathrm{~s}^{-1}$ but the power-law spectral index remains unconstrained. Its flux does not change over all the VPs in which it has been detected and it is a stationary gamma-ray source (see Fig. 1). The quite low upper limits $<10 \times 10^{-8} \mathrm{~cm}^{-2} \mathrm{~s}^{-1}$ found in other independent VPs have all $(T S)^{1 / 2}=0$ and can not be considered as statistically significant. The other reported upper limits on this source are consistent with the detection fluxes.

The Abell cluster Abell 3164 falls within the 95\% confidence level position error contours of the EGRET source. Also, there is no other identified gamma-ray source counterpart for this EGRET source. The EGRET source map is relatively broad with $\theta_{95}=0.42 \mathrm{deg}$ and has a comet-like tail in the east direction.
Abell 3164 is an irregular cluster with a redshift of $z=$ 0.057 (Struble et al. 1999) for which there is poor information available. Ebeling et al. (1996) estimated its X-ray luminosity to be $L_{0.5-2.4 \mathrm{keV}} \approx 1.48 \times 10^{44} \mathrm{erg} \mathrm{s}^{-1}$ and its temperature as $k T \approx 3.6 \mathrm{keV}$ from the ROSAT data. There are no evidence for NVSS radio sources found in correlation with this cluster, given the low cluster declination. A few SUMSS radio sources are found at more than $\sim 20$ arcmin from the cluster center.

Based on the previous evidence, we do not find any strong hint for the probable association of this cluster with the EGRET source 3EG J0348-5708 and we consider also this case as likely due to projection effects.

\subsection{EG J0159-3603}

This EGRET source has been detected with a $(T S)^{1 / 2}=4.3$ in the $\mathrm{VP}=\mathrm{P} 1234$. It has a flux $F_{\mathrm{P} 1234}(>100 \mathrm{MeV})=(9.8 \pm 2.8) \times$ $10^{-8} \mathrm{~cm}^{-2} \mathrm{~s}^{-1}$ with a power-law spectral index $\gamma=2.89 \pm 0.51$. Its flux does not change over all the VPs and it is a stationary gamma-ray source (see Fig. 1). Two Abell clusters fall within the $95 \%$ confidence level position error contours of this source: Abell 219S and Abell 2963. Other galaxy clusters are found in the vicinities of the EGRET source (see Fig. 5). The EGRET source map is quite broad with $\theta_{95}=0.79$ deg and the elongation of the EGRET probability map is aligned with the position of the two clusters, suggesting a possible contribution to the gamma-ray emission from both clusters. There is no other known gamma-ray source counterpart for this EGRET source.

There is little optical and X-ray information on both the clusters Abell 219S and Abell 2963. There are nonetheless three NVSS radio sources associated with the cluster Abell 2963: they have radio flux at $1.4 \mathrm{GHz}$ of $S_{1.4}=(23.44 \pm$ $1.57),(4.46 \pm 0.45)$ and $(2.43 \pm 0.45) \mathrm{mJy}$, respectively.

In view of these evidence, we consider that this is a candidate for a probable association between galaxy clusters and an EGRET gamma-ray source.

\subsection{EG J0616-3310}

This EGRET source has been detected with a $(T S)^{1 / 2}=4.7$ in the $\mathrm{VP}=\mathrm{P} 1234$. It has a flux $F_{\mathrm{P} 1234}(>100 \mathrm{MeV})=(12.6 \pm$ 3.2) $\times 10^{-8} \mathrm{~cm}^{-2} \mathrm{~s}^{-1}$ with a power-law spectral index $\gamma=$ $2.11 \pm 0.24$. Its flux changes significantly over several VPs and in the $\mathrm{VP}=419.5$ it increase by a factor $\gtrsim 4$ with respect to the $\mathrm{VP}=\mathrm{P} 1234$. Due to such strong flux variations in comparison with other cases shown in Fig. 1, it is hard to consider it as a stationary gamma-ray source.

Two clusters (Abell 577S and Abell 575S) fall close to the $95 \%$ confidence level error contour for the position of this EGRET source. Another cluster (Abell 573S) falls within $1 \mathrm{deg}$ radius from the center of the EGRET source. However, the shape of the EGRET map of this source is quite compact and round with $\theta_{95}=0.13 \mathrm{deg}$.

The two clusters Abell 577S and Abell 575S have very few morphological and physical information (see Table 1). Nonetheless, the cluster Abell 577S is associated with three NVSS radio sources with flux $S_{1.4}=(4.32 \pm 0.48)$, $(14.92 \pm 0.61)$ and $(12.48 \pm 1.57) \mathrm{mJy}$, respectively. Also 
Abell 575S is correlated with other three NVSS radio sources with flux $S_{1.4}=(5.86 \pm 0.48),(14.17 \pm 0.59)$ and $(3.03 \pm$ $0.47) \mathrm{mJy}$, respectively. Note that also the cluster Abell $573 \mathrm{~S}$ is associated with 7 NVSS radio sources.

Due to the previous evidence, and in particular the flux variation over the various VPs, we consider this association as suspect and probably due to projection effects.

\subsection{EG J2034-3110}

This EGRET source has been detected with a $(T S)^{1 / 2}=4.0$ in the $\mathrm{VP}=\mathrm{P} 1$. It has a flux $F_{\mathrm{P} 1}(>100 \mathrm{MeV})=(17.4 \pm 5.2) \times$ $10^{-8} \mathrm{~cm}^{-2} \mathrm{~s}^{-1}$ with a power-law spectral index $\gamma=3.43 \pm 0.78$. Even though the flux variations over the different VPs and the upper limit of $<6.2 \times 10^{-8} \mathrm{~cm}^{-2} \mathrm{~s}^{-1}$ with $(T S)^{1 / 2}=0$ found in the $\mathrm{VP}=209.0$ are not statistically significant, the behaviour of this EGRET source is quite different from the other sources here selected as possible association with galaxy clusters, which are expected to be quite stationary over different VPs. Due to such flux variations in comparison with other cases shown in Fig. 1, we do not consider it as a stationary gamma-ray source.

Nonetheless, this EGRET source is quite broad and irregular with a quite large value of $\theta_{95}=0.73 \mathrm{deg}$. The cluster Abell 886S falls within the $95 \%$ confidence level position error contour of the source. There is no other gamma-ray source counterpart in the field of 3EG J2034-3110.

The cluster Abell 886S has no detailed information available, it is not associated with any NVSS radio sources and there are no other hints for the presence of galaxy activity in its environment.

Due to the previous evidence, and in particular the flux variation over the various VPs, we consider also this association as suspect and probably due to projection effects.

\subsection{EG J1234-1318}

This EGRET source has been detected with a $(T S)^{1 / 2}=4.8$ in the $\mathrm{VP}=\mathrm{P} 1234$. It has a flux $F_{\mathrm{P} 1234}(>100 \mathrm{MeV})=(7.3 \pm$ 1.7) $\times 10^{-8} \mathrm{~cm}^{-2} \mathrm{~s}^{-1}$ with a quite low power-law spectral index $\gamma=2.09 \pm 0.24$. The flux variations over the different VPs are not statistically significant, and for this reason it can be considered as a stationary gamma-ray source. The upper limit of $<8.9 \times 10^{-8} \mathrm{~cm}^{-2} \mathrm{~s}^{-1}$ has $(T S)^{1 / 2}=0$ and its very poor statistical significance does not affect strongly the previous conclusion.

Two galaxy clusters (Abell 1558 and Abell 1555) fall within the $95 \%$ confidence level position error contour of the EGRET source. This EGRET source is quite regular with $\theta_{95}=0.76$ deg even though source confusion may affect its flux and/or its position (see Hartman et al. 1999). No other known gamma-ray source counterpart has been found in the field of this EGRET source.

Abell 1558 has an estimated redshift of $z=0.116$ (Andernach 2002, priv. comm.) and it is associated with two NVSS radio sources with flux $S_{1.4}=2.92 \pm 0.47$ and $7.73 \pm 1.24 \mathrm{mJy}$, respectively. Also the cluster Abell 1555 at $z=0.127$ is associated with two NVSS radio sources with flux $S_{1.4}=7.14 \pm 0.48$ and $4.24 \pm 0.48 \mathrm{mJy}$, respectively.
No other information is available on these clusters at both optical and X-ray frequencies.

Due to the previous evidence, we find that the association of this EGRET source with the two Abell clusters here mentioned is still questionable.

\subsection{EG J0038-0949}

This EGRET source has been detected with a $(T S)^{1 / 2}=4.1$ in the $\mathrm{VP}=\mathrm{P} 1234$. It has a flux $F_{\mathrm{P} 1234}(>100 \mathrm{MeV})=(12.0 \pm 3.7) \times$ $10^{-8} \mathrm{~cm}^{-2} \mathrm{~s}^{-1}$ with a power-law spectral index $\gamma=2.70 \pm 0.44$. The flux variations over the different VPs are at less than the $2 \sigma$ level and so are not strongly statistically significant. Also the low upper limit of $<11.8 \times 10^{-8} \mathrm{~cm}^{-2} \mathrm{~s}^{-1}$ obtained in the $\mathrm{VP}=327.0$ has $(T S)^{1 / 2}=0$ and it is not statistically significant. However, the behaviour of the flux changes in the different viewing periods over which this EGRET source has been detected is somewhat different from a purely stationary source as shown in Fig. 1. This EGRET source is elongated in the east-west direction and has a value $\theta_{95}=0.59 \mathrm{deg}$.

The optical and X-ray center of the cluster Abell 85 is found slightly beyond the $95 \%$ confidence level position error contour of the EGRET source (see Fig. 4). However, due to its large extension ( $~ 230$ arcmin radius) a large part of this nearby $(z=0.056)$ cluster falls within the $95 \%$ confidence level position error contour of the EGRET source and hence can be considered to be spatially correlated with it. No other known gamma-ray source counterpart is found in the field of this EGRET source.

Abell 85 is a bright $\mathrm{X}$-ray cluster with a luminosity $L_{2-10 \mathrm{keV}}=(7.65 \pm 0.52) \times 10^{44} \mathrm{erg} \mathrm{s}^{-1}$ and a temperature of $k T=6.2 \pm 0.4 \mathrm{keV}$ (Wu et al. 1999) and shows a strong activity in its ICM.

In fact, there are several bright radio galaxies within the cluster Abell 85 and also several bright NVSS radio sources correlated with the cluster as well as in the field of the relative EGRET source. Abell 85 has been observed with the VLA in the B and C configurations and a flux $S_{1.4}=55 \mathrm{mJy}$ has been reported by Owen \& Ledlow (1997). Abell 85 contains also a diffuse, relic radio source (see, e.g., Slee et al. 2001) found off-center with respect to the X-ray center of the cluster (see Fig. 6). Giovannini \& Feretti (2000) estimated that the diffuse radio halo flux at $1.4 \mathrm{GHz}$ is $S_{1.4}=46 \mathrm{mJy}$, consistently with the result of Owen \& Ledlow (1997), with a power $P_{1.4}=6.1 \times$ $10^{23} \mathrm{~W} \mathrm{~Hz}^{-1}$.

There is also evidence of a hard X-ray emission excess which is spatially correlated with the radio relic source and is due probably to Inverse Compton Scattering (ICS) of the CMB photons with the relativistic electrons of the radio relic (Bagchi et al. 1998; Lima-Neto et al. 2001). Such a non-thermal X-ray emission is spatially correlated with the Very Steep Spectrum radio source MRC 0038-096 (see Bagchi et al. 1998), without any detected optical counterpart, which is $\sim 7$ arcmin southwest of the X-ray center of the cluster.

The positive detection of both synchrotron radio and ICS X-ray emission from a common ensemble of relativistic electrons leads to an estimate of the average magnetic field, 


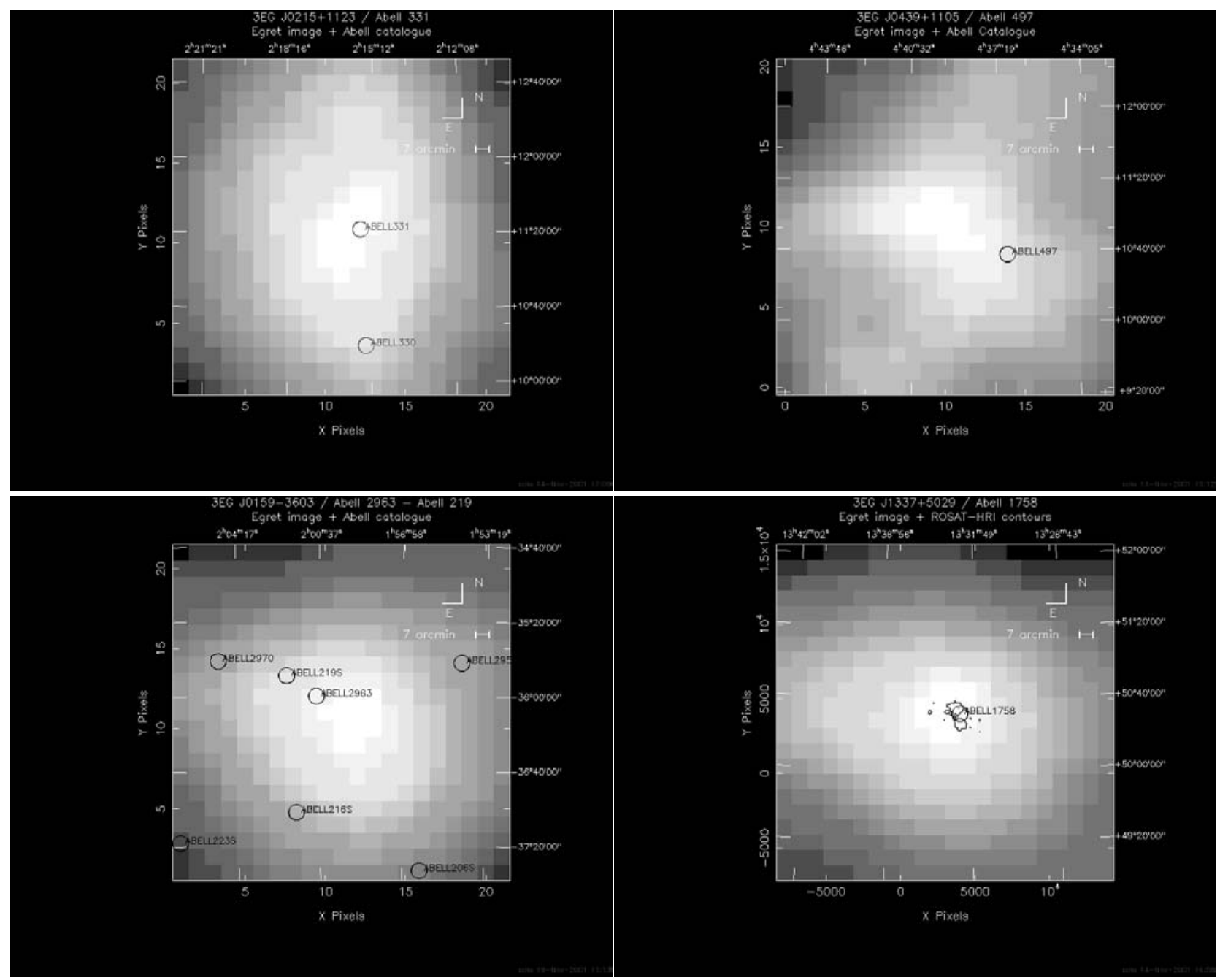

Fig. 5. We show here the positions of the galaxy clusters which are probable candidates for the associations with unidentified EGRET sources: 3EGJ0215+1123 associated with Abell 331 (upper left), 3EGJ0439+1105 associated with Abell 497 (upper right), 3EGJ0159-3603 associated with Abell 2963 and Abell 219S (lower left) and 3EGJ1337+5029 associated with Abell 1758 (lower right). The cluster positions and the X-ray brightness contours (when available) are superposed to the maps of the EGRET sources. The intensity scale of the EGRET maps goes from black (minimum) to white (maximum).

$B \approx 0.95 \pm 0.10 \mu \mathrm{G}$, on the cluster scale. Further, the radiative flux and the estimated value of $B$ imply the presence of relativistic electrons (with radiative lifetime $\gtrsim 10^{9} \mathrm{yr}$ ) with Lorentz factor $\gamma_{\mathrm{L}} \approx 700-1700$ (Bagchi et al. 1998). Electrons with these energies can easily emit gamma-rays at $E_{\gamma}>100 \mathrm{MeV}$ by bremsstrahlung in addition to the ICS emission tail which is present in the gamma-ray region probed by EGRET.

Even though the cluster Abell 85 is offset with respect to the center of the EGRET source map, there are good reasons to believe that it may contribute substantially to the gammaray flux of the EGRET source 3EG J0038-0949 in addition to the possible gamma-ray flux possibly produced by the active radio-galaxies which are living in the cluster environment.

\section{9. $3 E G$ J1310-0517}

This EGRET source has been detected with a $(T S)^{1 / 2}=5.0$ in the $\mathrm{VP}=\mathrm{P} 1234$. It has a flux $F_{\mathrm{P} 1234}(>100 \mathrm{MeV})=(7.9 \pm 1.8) \times$ $10^{-8} \mathrm{~cm}^{-2} \mathrm{~s}^{-1}$ with a power-law spectral index $\gamma=2.34 \pm 0.22$. The flux variations over the different VPs are at less than the
$2 \sigma$ level and are not statistically significative (see Fig. 1). Also the lowest upper limit $<10.9 \times 10^{-8} \mathrm{~cm}^{-2} \mathrm{~s}^{-1}$, obtained for this source in the $\mathrm{VP}=$ Virgo4 with $(T S)^{1 / 2}=0.5$ has a very poor statistical significance. The EGRET source is elongated in the south-north direction and has a value $\theta_{95}=0.78 \mathrm{deg}$.

The center of the cluster Abell 1688 is found within the $95 \%$ confidence level position error contour of the source, even though quite off-center with respect to the EGRET map center (see Fig. 4). There is, however, no other known gamma-ray source counterpart in the field of this EGRET source.

Abell 1688 is one of the most distant clusters listed in Table 1 and has little information available at both optical and X-ray wavelengths. Kowalski et al. (1984) gave an estimate of its redshift $z \sim 0.19$ and of its X-ray luminosity, $L_{2-10 \mathrm{keV}} \lesssim 4.79 \times 10^{44} \mathrm{erg} \mathrm{s}^{-1}$ as obtained from the HEAO-A1 all-sky survey. There are, nonetheless, 4 NVSS radio sources correlated with the position of Abell 1688 and they have flux $S_{1.4}=8.63 \pm 0.50 ; 59.83 \pm 2.25 ; 10.29 \pm 0.53 ; 3.66 \pm 0.51 \mathrm{mJy}$, respectively. 


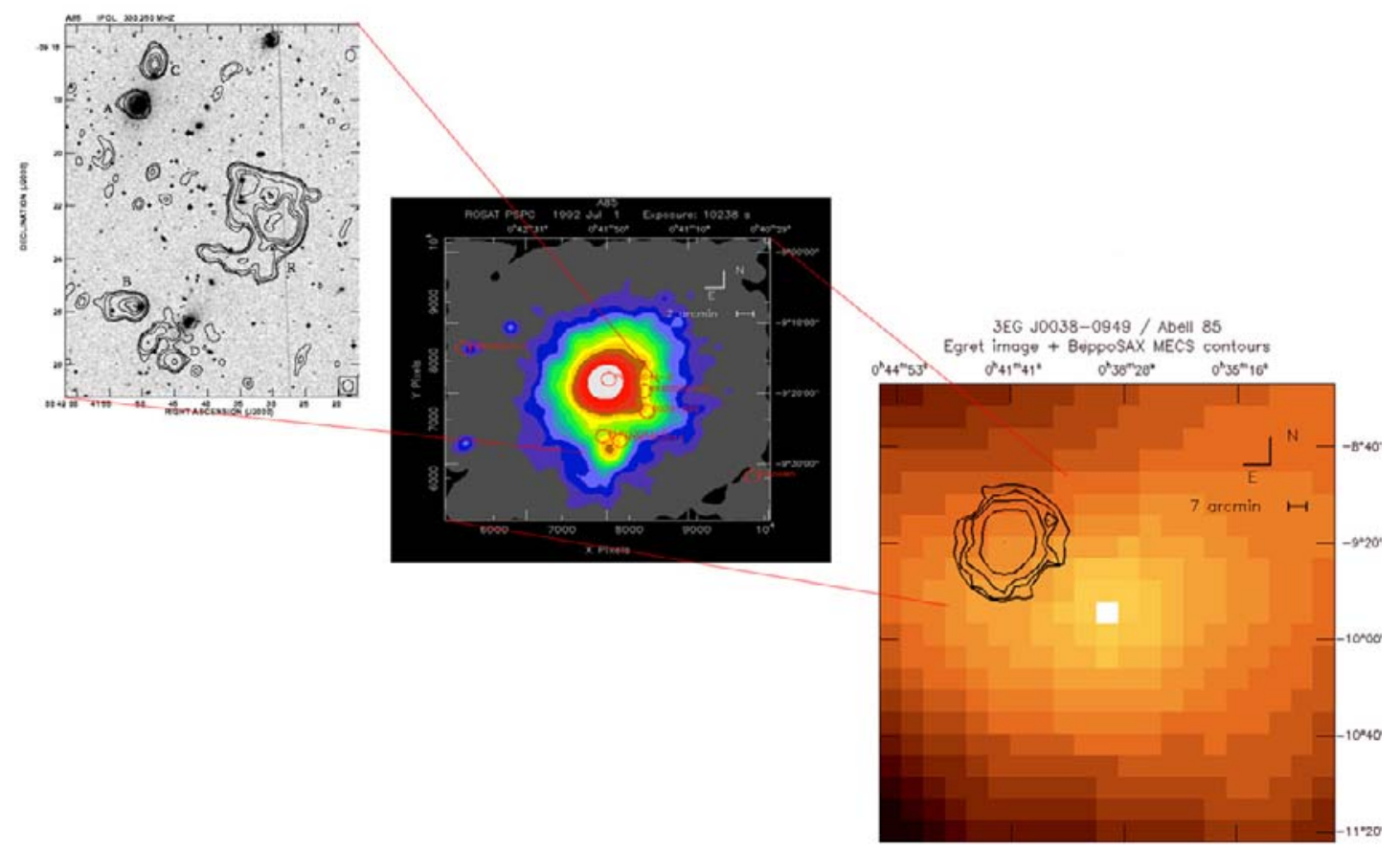

Fig. 6. One of the most probable associations between galaxy clusters and EGRET unidentified gamma-ray sources: Abell 85 . This cluster has a radio halo/relic inhabiting the cluster and a number of identified radio galaxies. Shown are the EGRET image with the cluster X-ray brightness contours (right), the ROSAT-HRI X-ray image of the cluster (center) with the NVSS radio sources in the field (red circles) and the radio halo/relic image obtained with the VLA at $327 \mathrm{MHz}$ (left).

Due to these evidence, this could be considered as a probable - but still questionable - association between galaxy clusters and EGRET gamma-ray source.

\subsection{EG J0253-0345}

This EGRET source has been detected with a $(T S)^{1 / 2}=4.0$ in the $\mathrm{VP}=317.0$. It has a flux $F_{317.0}(>100 \mathrm{MeV})=(17.3 \pm 5.7) \times$ $10^{-8} \mathrm{~cm}^{-2} \mathrm{~s}^{-1}$ with a power-law spectral index which is unconstrained. There are no flux variations over the two VPs in which the source has been detected (see Fig. 1). The low upper limit $<4.2 \times 10^{-8} \mathrm{~cm}^{-2} \mathrm{~s}^{-1}$ derived in the $\mathrm{VP}=21.0$ has $(T S)^{1 / 2}=0$ and is not statistically significant. The EGRET source map is quite extended and round with a high value of $\theta_{95}=1.13 \mathrm{deg}$.

The cluster Abell 388 falls within the $95 \%$ confidence level position error contour of the source (see Fig. 4) and other Abell clusters are found in the field of this EGRET source. No other possible counterpart of the gamma-ray source is found in the field of this EGRET source.

Abell 388 has a redshift of $z=0.134$ and an estimated Xray luminosity of $L_{2-10 \mathrm{keV}} \lesssim 3.47 \times 10^{44} \mathrm{erg} \mathrm{s}^{-1}$, as reported in the HEAO-A1 all-sky survey (Kowalski et al. 1984). No other relevant X-ray information is available for this cluster. There are 8 NVSS radio sources correlated with this cluster with flux $S_{1.4}=8.42 \pm 0.50,4.69 \pm 0.47,10.08 \pm 1.13,4.18 \pm 0.48,7.58 \pm$ $0.49,15.32 \pm 1.13,3.91 \pm 0.48$ and $4.03 \pm 0.48 \mathrm{mJy}$, respectively.

Due to these evidence, this could be considered as a probable - but not yet definite - association between galaxy clusters and EGRET gamma-ray source.

\subsection{EG J0439+1105}

This EGRET source has been detected with a $(T S)^{1 / 2}=4.2$ in the $\mathrm{VP}=\mathrm{P} 1234$. It has a flux $F_{\mathrm{P} 1234}(>100 \mathrm{MeV})=(9.4 \pm 2.4) \times$ $10^{-8} \mathrm{~cm}^{-2} \mathrm{~s}^{-1}$ with a power-law spectral index $\gamma=2.44 \pm 0.29$. There are no statistically significative flux variations over the different VPs in which the source has been detected (see Fig. 1) and the statistically significant upper limits are consistent with this conclusion. The EGRET source error area is quite extended with a high value of $\theta_{95}=0.92 \mathrm{deg}$.

The cluster Abell 497 falls within the 95\% confidence level position error contour of the source. No other gamma-ray source counterpart is found in the field of this EGRET source (see Fig. 5).

Abell 497 has an estimated redshift of $z \sim 0.14$ and an estimated X-ray luminosity of $L_{2-10 \mathrm{keV}} \sim 3.89 \times 10^{44} \mathrm{erg} \mathrm{s}^{-1}$ (Ulmer et al. 1980). There is a NVSS radio source correlated with the cluster within its Abell radius with a flux $S_{1.4}=3.27 \pm$ 0.47 mJy.

Due to these evidence, this could be considered as a probable - but not yet definite - association between galaxy clusters and EGRET gamma-ray source.

\subsection{2. $3 E G$ J0215+1123}

This EGRET source has been detected with a $(T S)^{1 / 2}=4.4$ in the $\mathrm{VP}=21.0$. It has a flux $F_{21.0}(>100 \mathrm{MeV})=(18.0 \pm$ $5.0) \times 10^{-8} \mathrm{~cm}^{-2} \mathrm{~s}^{-1}$ with a quite low power-law spectral in$\operatorname{dex} \gamma=2.03 \pm 0.62$. There are no other definite detection of this EGRET source over other viewing periods (see Fig. 1). The upper limit of $6.0 \times 10^{-8} \mathrm{~cm}^{-2} \mathrm{~s}^{-1}$ obtained in the $\mathrm{VP}=317.0$ is 
not statistically significant since it has $(T S)^{1 / 2}=0$. The EGRET source is quite extended with a high value of $\theta_{95}=1.06 \mathrm{deg}$ and is elongated in the south-north direction.

The cluster Abell 331 falls near the center of the EGRET source (see Fig. 5) and there is no other gamma-ray source counterpart in the field of 3EG J0215+1123. There is another Abell cluster (Abell 330) which is found at $\sim 1.1 \mathrm{deg}$ south of Abell 331. The cluster Abell 331 has an estimated redshift of $z=0.186$ and an X-ray luminosity of $L_{2-10 \mathrm{keV}} \lesssim$ $5.01 \times 10^{44} \mathrm{erg} \mathrm{s}^{-1}$ as estimated in the HEAO-A1 all-sky survey (Kowalski et al. 1984). There are four NVSS radio sources which are correlated with this cluster within its Abell radius and their flux is $S_{1.4}=3.68 \pm 0.48 ; 3.38 \pm 0.48 ; 4.73 \pm 0.49$; $133.93 \pm 4.42 \mathrm{mJy}$, respectively.

Due to these evidence, this could be considered as a probable - but still questionable - association between galaxy clusters and EGRET gamma-ray source.

\subsection{EG J2248+1745}

This EGRET source has been detected with a $(T S)^{1 / 2}=4.1$ in the $\mathrm{VP}=\mathrm{P} 1234$. It has a flux $F_{\mathrm{P} 1234}(>100 \mathrm{MeV})=(12.9 \pm$ $3.5) \times 10^{-8} \mathrm{~cm}^{-2} \mathrm{~s}^{-1}$ with a power-law spectral index $\gamma=2.11 \pm$ 0.39 . The flux variations over the different VPs are at less than the $2 \sigma$ level, but the behaviour of this source is quite different from the stationary ones in Fig. 1. The EGRET source is quite extended with a value $\theta_{95}=0.94 \mathrm{deg}$ and is elongated in the south-north direction.

The cluster Abell 2486 falls within the 95\% confidence level position error contour of the source. No other gamma-ray source counterpart is found in the field of this EGRET source.

Abell 2486 has an estimated redshift of $z=0.143$ and an $\mathrm{X}$-ray luminosity of $L_{2-10 \mathrm{keV}} \lesssim 1.48 \times 10^{44} \mathrm{erg} \mathrm{s}^{-1}$ as estimated in the HEAO-A1 all-sky survey (Kowalski et al. 1984). There are two NVSS radio sources which are correlated with this cluster within its Abell radius and their flux is $S_{1.4}=4.60 \pm 0.51$ and $4.84 \pm 0.49 \mathrm{mJy}$, respectively.

Due to these evidence, and in particular to the flux variations over the various VPs, this case should not be considered as a possible association between galaxy clusters and an EGRET gamma-ray source.

\subsection{EG J1212+2304}

This EGRET source has been detected with a $(T S)^{1 / 2}=3.3$ in the VP=Virgo2. It has a flux $F_{\text {Virgo2 }}(>100 \mathrm{MeV})=(19.7 \pm$ 7.7) $\times 10^{-8} \mathrm{~cm}^{-2} \mathrm{~s}^{-1}$ with a power-law spectral index $\gamma=$ $2.76 \pm 0.60$. Even though the flux of this source may be affected by confusion, there are quite strong flux variations over the three different VPs in which this source has been detected (see Fig. 1). The EGRET source is quite extended with a value $\theta_{95}=0.88 \mathrm{deg}$ and is elongated in the south-north direction.

The cluster Abell 1494 falls within the 95\% confidence level position error contour of the source. No other gamma-ray source counterpart is found in the field of this EGRET source.

Abell 1494 has an estimated redshift of $z=0.159$ and an X-ray luminosity of $L_{2-10 \mathrm{keV}} \lesssim 1.95 \times 10^{45} \mathrm{erg} \mathrm{s}^{-1}$ as estimated in the HEAO-A1 all-sky survey (Kowalski et al. 1984). There are five NVSS radio sources which are correlated with this cluster and their flux is $S_{1.4}=4.91 \pm 0.48 ; 8.05 \pm 0.49$; $23.68 \pm 1.74 ; 17.53 \pm 0.66 ; 5.15 \pm 0.47 \mathrm{mJy}$, respectively.

Due to these evidence, and in particular to the flux variations over the various VPs, this case should not be considered as a possible association between galaxy clusters and EGRET gamma-ray source.

\subsection{5. $3 E G ~ J 1347+2932$}

This EGRET source has been detected with a $(T S)^{1 / 2}=4.0$ in the $\mathrm{VP}=\mathrm{P} 1234$. It has a flux $F_{\mathrm{P} 1234}(>100 \mathrm{MeV})=(9.6 \pm 2.9) \times$ $10^{-8} \mathrm{~cm}^{-2} \mathrm{~s}^{-1}$ with a power-law spectral index $\gamma=2.51 \pm 0.61$. There are no strong flux variations over the different VPs in which this source has been detected (see Fig. 1), even though the flux of this source may be affected by confusion. However, we noticed that there is only one independent detection of this source in the VP=4.0 which does not allow to draw any definite conclusion on its possible variability. The EGRET source is quite extended with a value of $\theta_{95}=0.95 \mathrm{deg}$ and is irregular.

The cluster Abell 1781 falls within the 95\% confidence level position error contour of the source. No other gamma-ray source counterpart is found in the field of this EGRET source.

Abell 1781 has a redshift of $z=0.0618$ and an X-ray luminosity of $L_{2-10 \mathrm{keV}} \sim 1.15 \times 10^{44} \mathrm{erg} \mathrm{s}^{-1}$ as estimated in the HEAO-A1 all-sky survey (Kowalski et al. 1984). There is one radio galaxy (FIRST J134159.7+294653) at a redshift of $z=0.0457$ which is apparently in the foreground of the cluster. There is evidence for 36 NVSS radio sources correlated with Abell 1781 within its Abell radius.

Due to these evidence, and in particular to the uncertainties in the flux variations over the various VPs, this case should not be considered as a possible association between galaxy clusters and EGRET gamma-ray source.

\subsection{6. $3 E G ~ J 1424+3734$}

This EGRET source has been detected with a $(T S)^{1 / 2}=4.4$ in the $\mathrm{VP}=\mathrm{P} 1$. It has a flux $F_{\mathrm{P} 1}(>100 \mathrm{MeV})=(16.3 \pm 4.9) \times$ $10^{-8} \mathrm{~cm}^{-2} \mathrm{~s}^{-1}$ with a power-law spectral index $\gamma=3.25 \pm 0.46$. There are no strong flux variations over the different VPs in which this source has been detected (see Fig. 1) and the lowest upper limit of $<16.1 \times 10^{-8} \mathrm{~cm}^{-2} \mathrm{~s}^{-1}$ found in the VP=201.+ is not statistically significant because it has $(T S)^{1 / 2}=0$. The EGRET source is quite regular with a value $\theta_{95}=0.88 \mathrm{deg}$ and with an emission tail in the north-west side of the field.

Two rich clusters (Abell 1914 and Abell 1902) fall within the $95 \%$ confidence level position error contour of the source (see Fig. 4). No other gamma-ray source counterpart is found in the field of this EGRET source.

Abell 1902 has a redshift of $z=0.16$ and is associated with the X-ray source RXJ1421.6+3717 (Boehringer et al. 2000) with an X-ray flux $F_{0.1-2.4 \mathrm{keV}}=(4.3 \pm 10.8) \times$ $10^{-12} \mathrm{erg} \mathrm{cm}^{-2} \mathrm{~s}^{-1}$ and a luminosity $L_{0.1-2.4 \mathrm{keV}} \sim 6 \times$ $10^{44} \mathrm{erg} \mathrm{s}^{-1}$. Its X-ray luminosity has been also estimated to be $L_{2-10 \mathrm{keV}} \sim 1.26 \times 10^{44} \mathrm{erg} \mathrm{s}^{-1}$ in the HEAO-A1 allsky survey (Kowalski et al. 1984). There is one radio source (FIRST J142140.4+371731) associated to the cluster galaxy 
MAPS-NGP 0-272-0323568 found at a redshift of $z=0.16$. There is also a NVSS radio source with flux $S_{1.4}=3.51 \pm$ $0.46 \mathrm{mJy}$ which is associated with the cluster.

Abell 1914 has a redshift of $z=0.1712$ and is associated to the X-ray source RXJ1426.0+3749 with an Xray flux $F_{0.1-2.4 \mathrm{keV}}=(12.90 \pm 5.2) \times 10^{-12} \mathrm{erg} \mathrm{cm}^{-2} \mathrm{~s}^{-1}$. Its X-ray luminosity has been estimated to be $L_{0.1-2.4 \mathrm{keV}} \sim$ $15.91 \times 10^{44} \mathrm{erg} \mathrm{s}^{-1}$ (Boehringer et al. 2000). Three NVSS radio sources are correlated with Abell 1914 and they have flux $S_{1.4}=9.91 \pm 1.50,30.86 \pm 1.77,20.75 \pm 4.08 \mathrm{mJy}$, respectively. The cluster Abell 1914 also hosts the Very Steep Spectrum radio galaxy 1474+380 (4C 38.39) (Komissarov \& Gubanov 1994). This cluster has also a bright radio halo (see Fig. 7) detected in the VLA with a flux of $S_{1.4}=50 \mathrm{mJy}$ and a power $P_{1.4}=6.31 \times 10^{24} \mathrm{~W} \mathrm{~Hz}^{-1}$ (Giovannini \& Feretti 2000).

Due to the previous evidence we consider this case as a probable candidate for the correlation of galaxy clusters and EGRET unidentified gamma-ray sources.

\subsection{7. $3 E G ~ J 1337+5029$}

This EGRET source has been detected with a $(T S)^{1 / 2}=4.4$ in the $\mathrm{VP}=\mathrm{P} 1234$. It has a flux $F_{\mathrm{P} 1234}(>100 \mathrm{MeV})=(9.2 \pm 2.6) \times$ $10^{-8} \mathrm{~cm}^{-2} \mathrm{~s}^{-1}$ with a power-law spectral index $\gamma=1.83 \pm 0.29$. There are no strong flux variations over the different VPs in which this source has been detected (see Fig. 1). The EGRET source is quite regular with a value $\theta_{95}=0.72 \mathrm{deg}$.

The rich cluster Abell 1758 falls within the $95 \%$ confidence level position error contour of the source, very close to the center of the EGRET source map (see Fig. 5). No other gamma-ray source counterpart is found in the field of this EGRET source.

Abell 1758 is the most distant cluster listed in Table 1. It has a redshift of $z=0.279$ and is associated with the X-ray source RXJ1332.7+5032 with an X-ray flux $F_{0.1-2.4 \mathrm{kev}}=(5.6 \pm 9.5) \times 10^{-12} \mathrm{erg} \mathrm{cm}^{-2} \mathrm{~s}^{-1}$ and a luminosity $L_{0.1-2.4 \mathrm{keV}} \sim 1.8 \times 10^{45} \mathrm{erg} \mathrm{s}^{-1}$ (Boehringer et al. 2000). The ROSAT-PSPC observation yielded a temperature $k T \approx 4.1 \mathrm{keV}$ which is found to be much lower than the ASCA (SIS+GIS) temperature of $T \approx 9.33 \mathrm{keV}$ (Rizza et al. 1995). An X-ray luminosity of $L_{2-10 \mathrm{keV}} \approx(1.43 \pm 0.06) \times 10^{45} \mathrm{erg} \mathrm{s}^{-1}$ has been estimated independently by Wu et al. (1999).

There are four NVSS radio sources correlated with this cluster with a flux $S_{1.4}=109.83 \pm 3.86,8.69 \pm 1.42$, $14.83 \pm 1.51,5.18 \pm 0.43 \mathrm{mJy}$, respectively. Abell 1758 also hosts the narrow tailed radio galaxy 87GB $133050.3+504752$ (Feretti et al. 1992). This cluster also shows a diffuse radio emission (see Fig. 8) which could be possibly identified with an extended radio halo (Giovannini \& Feretti 2000).

Due to the previous evidence we consider this case as a probable candidate for the correlation of galaxy clusters and EGRET unidentified gamma-ray sources.

\subsection{EG J1447-3936}

This EGRET source has been detected with a $(T S)^{1 / 2}=4.5$ in the $\mathrm{VP}=\mathrm{P} 1234$. It has a flux $F_{\mathrm{P} 1234}(>100 \mathrm{MeV})=(11.0 \pm$ 2.7) $\times 10^{-8} \mathrm{~cm}^{-2} \mathrm{~s}^{-1}$ with a power-law spectral index $\gamma=2.45 \pm$ 0.34 . There are no strong flux variations over the different VPs in which this source has been detected (see Fig. 1). However, the only two independent detections of this source do not allow to draw any definite conclusion on its variability. The EGRET source is quite regular with a value $\theta_{95}=0.87 \mathrm{deg}$.

The cluster Abell $774 \mathrm{~S}$ falls within the 95\% confidence level position error contour of the source. No other gamma-ray source is found in the field of this EGRET source.

Abell $774 \mathrm{~S}$ is a poor cluster with very limited information at other wavelengths. There are 9 NVSS radio sources correlated with this cluster within its Abell radius. There are also 2 PMN sources within the Abell radius of Abell 774S with flux $S_{4.85}=126 \pm 11 \mathrm{mJy}$ and $67 \pm 10 \mathrm{mJy}$, respectively. The latter one coincides with NVSS J144952.4-395732 with $S_{1.4}=55 \mathrm{mJy}$, an inverted-spectrum radio source.

We thus believe that this case of spatial correlation is likely due to projection effects.

\section{The gamma-ray - radio correlation}

Many of the clusters listed in Table 1 (namely, 17 out of 24 clusters) have also NVSS and SUMSS radio sources within their Abell radius, five clusters have identified bright radio galaxies in their environment and three clusters (Abell 1758, Abell 1914 and Abell 85) have also a radio halo or radio relic inhabiting their ICM (see Figs. 6-8). Since the EGRET sources have been selected to be of high galactic latitude, the NVSS radio sources are very likely non-identified (active) radio galaxies, as also indicated by the NVSS-NRAO images of many of the radio sources found in our analysis of the specific EGRET sources counterparts as discussed in Sect. 3 above.

The 9 EGRET sources in Table 1 which are marked with an asterisk are those more likely associated to galaxy clusters according to our analysis of the specific sources presented in Sect. 3 above. These galaxy clusters are quite peculiar since all of them have bright NVSS radio sources in their environment and six of them have also bright radio galaxies living in their environment. Three of the clusters which are more probably associated with these EGRET sources show also the presence of extended radio halos or relics. Hence, such galaxy clusters which have strong radio emission (either diffuse or associated with member galaxies) show the direct presence of a population of relativistic electrons which are injected in their ICM.

Radio galaxies, which are mainly found in the central regions of the clusters, may inject into the cluster ICM large quantities of energy transported by their relativistic jets. This energy is probably changed from an electromagnetic form to a pair plasma, to an ion plasma and (at least partially along the way) into energetic photons (see, e.g., Blandford 2002). Such high-energy particles and photons may produce $\sim \mathrm{GeV}$ gamma-ray emission which can be observed by EGRET. The active galaxy radio power correlates with the gamma-ray power (Padovani et al. 1993) indicating that radio louder galaxies emit more gamma-ray power which, in turn, seems to be associated with relativistic beaming of the jets (see, e.g., Urry \& Padovani 1995). Also the particles injected into the ICM by the radio-galaxy jets may diffuse in the magnetized ICM (Colafrancesco \& Blasi 1998) and interact with the ICM particles (mainly electrons and protons) to produce diffuse radio emission (Blasi \& Colafrancesco 1999), heating of the ICM 


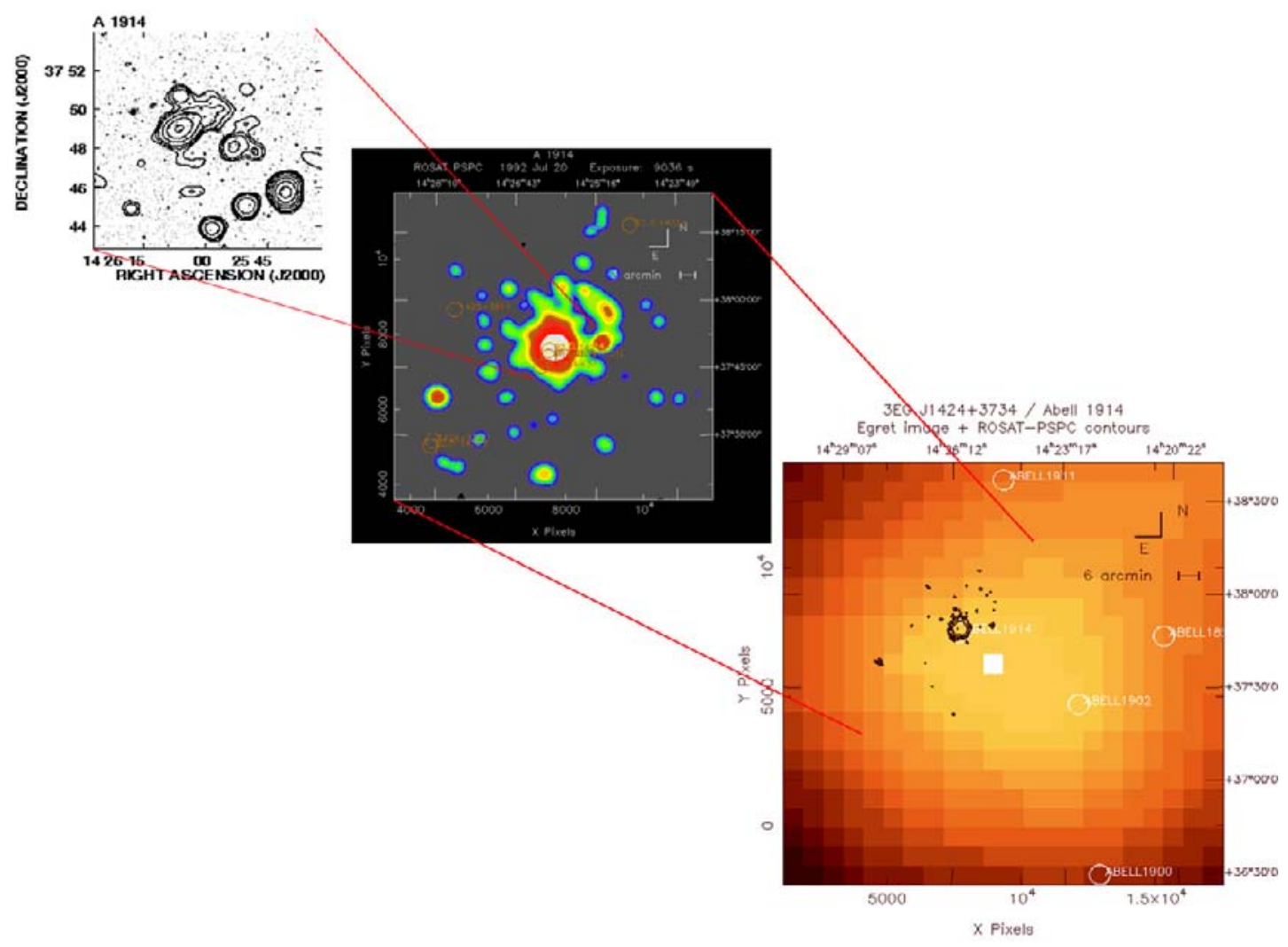

Fig. 7. One of the most probable associations between galaxy clusters and EGRET unidentified gamma-ray sources: Abell 1914. This cluster has a radio halo/relic inhabiting the cluster and a number of identified radio galaxies in the ICM. Shown are the EGRET source image with the cluster X-ray brightness contours (right), the ROSAT-HRI X-ray image of the cluster (center) with the NVSS radio sources in the field (red circles) and the radio halo/relic image observed with the VLA at $1.4 \mathrm{GHz}$ (left).

itself (Yamada \& Fujita 2001; Kaiser \& Alexander 1999; Inoue \& Sasaki 2001; Nath \& Roychowdhury 2002) and secondarily produced gamma-ray emission (Colafrancesco \& Blasi 1998; Blasi 2000).

In addition, particles in the ICM could be efficiently accelerated at the accretion shocks located at the cluster periphery as well as at the ICM shocks produced by subcluster mergings (see, e.g., Miniati et al. 2000) and/or by fast galaxy motions. The subsequent interaction of the accelerated particles with the surrounding hot, magnetized ICM can again produce diffuse radio halo/relic emission (see, e.g., Blasi \& Colafrancesco 1999; Sarazin 2002) and diffuse gamma-ray emission at $E>$ $100 \mathrm{MeV}$ (Colafrancesco \& Blasi 1998). On top of these acceleration mechanisms, it has been recently shown that dark matter particle (neutralinos) annihilation - a mechanism which is especially efficient in the central regions of the clusters - may produce both diffuse radio halo emission and diffuse gammaray emission visible at $E>100 \mathrm{MeV}$ (Colafrancesco \& Mele 2001).

The presence of such relativistic particles into the ICM strongly suggests, in conclusion, that themselves and/or their parent population (e.g., relativistic protons, dark matter particles) can be responsible for a substantial gamma-ray flux at the EGRET energies $(E>100 \mathrm{MeV})$ as well as non-thermal radio emission through different mechanisms. Thus, we propose that there should be a close connection between radio emission (either diffuse or associated with individual active galaxies) and gamma-ray emission in galaxy clusters.

Based on the previous arguments, we should expect a positive correlation between the flux of the radio sources associated with the galaxy cluster and the gamma-ray flux of the relative EGRET source. In fact, we found a positive correlation between the radio flux at $1.4 \mathrm{GHz}, S_{1.4}$, of the brightest radio source in the cluster and the EGRET source flux, $F(>100 \mathrm{MeV})$, which is reported in the Third EGRET Catalog (Hartman et al. 1999). Specifically, we find a correlation $F(>100 \mathrm{MeV})=A S_{1.4}^{B}$ with $A=6.053_{-1.836}^{+2.637}$ and $B=0.187 \pm 0.091$ ( $1 \sigma$ errors) using gamma-ray fluxes selected in the different observing periods of the EGRET source (see Fig. 9). The best fit has a $\chi^{2}=2.38$ which gives a probability $P=0.064$ for the null hypothesis of a random distribution for the radio and gamma-ray flux of the nine sources in our analysis. This gives a statistical confidence level of $\approx 2.05 \sigma$. We show in Fig. 9 the best fit curve and the $1 \sigma$ and $2 \sigma$ confidence level regions for the fit. A similar result obtains considering the total radio flux from the clusters (most of the clusters here considered have more than one radio source in their environment) associated with the previous EGRET sources. The reason for such similar result is that in many cases the cluster radio flux at $1.4 \mathrm{GHz}$ is dominated by the brightest radio source in the cluster. 


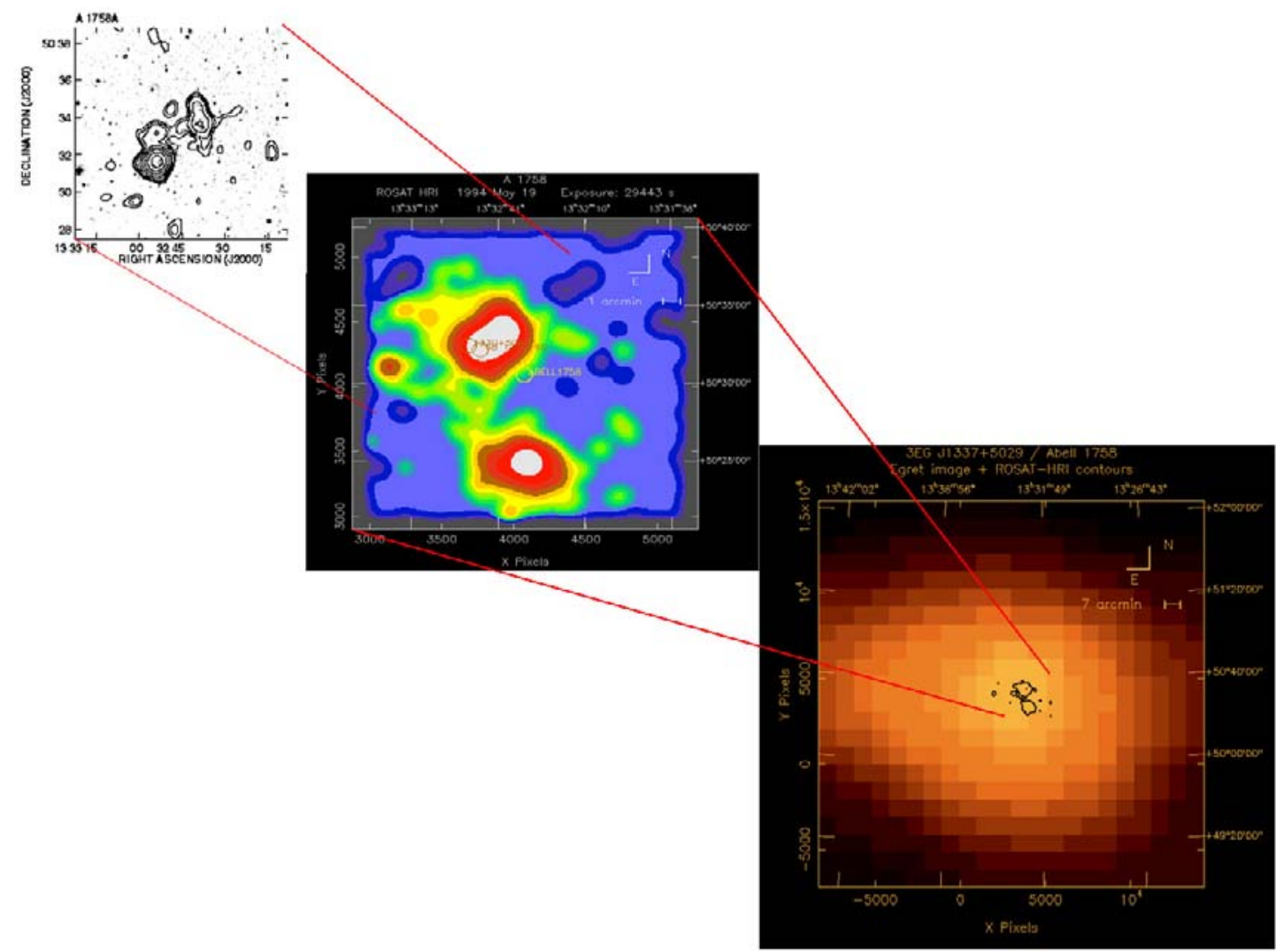

Fig. 8. One of the most probable associations between galaxy clusters and EGRET unidentified gamma-ray sources: Abell 1758. This cluster has a radio halo/relic inhabiting the cluster and a number of identified radio galaxies in the ICM. Shown are the EGRET image with the cluster X-ray brightness contours superposed (right), the ROSAT-HRI X-ray image of the cluster (center) with the NVSS radio sources in the field (red circles) and the radio halo/relic image observed with the VLA at $1.4 \mathrm{GHz}$ (left).

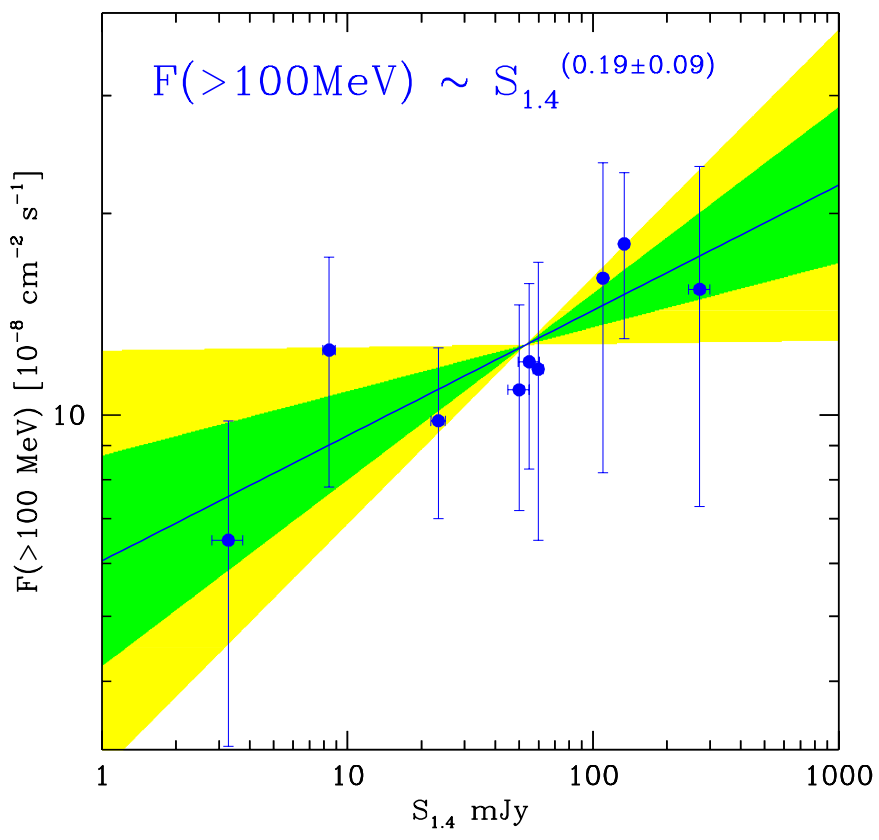

Fig. 9. The correlation between the gamma-ray flux, $F(>100 \mathrm{MeV})$, and the radio flux at $1.4 \mathrm{GHz}, S_{1.4}$, shown by the EGRET source - cluster associations listed with an asterisk in Table 1. The best fit curve $F(>100 \mathrm{MeV}) \sim S_{1.4}^{0.19}$ is shown (solid) together with the $1 \sigma$ (green/dark gray) and $2 \sigma$ (yellow/pale gray) confidence level regions of the fit.
Even though the large uncertainties in the EGRET source fluxes do not allow to draw any strong conclusion for the universality of such correlation, the present results indicate that there is a connection between the activity of the cluster ICM, and of its active galaxy content, and the overall gamma-ray behaviour of these large-scale structures, an indication that can be definitely confirmed by the next generation gamma-ray telescopes. The detailed follow-up of the galaxy populations of the clusters most probably associated to the 9 EGRET sources here selected is an important aspect in this research field but it is far beyond the aims of the present work and will be tackled in a forthcoming paper.

\section{The gamma-ray - X-ray luminosity correlation}

To further strengthen our argument presented in the previous sections, we looked for other intrinsic correlations among galaxy clusters and unidentified EGRET sources. Specifically, we correlated the cluster X-ray luminosity, $L_{\mathrm{X}}$, with the luminosity of the associated EGRET source under the assumption that it is physically associated to the cluster and, hence, has the same redshift. We derived the gamma-ray luminosity, $L_{\gamma}$, of each EGRET source from the gamma-ray fluxes at $E>100 \mathrm{MeV}$ given in the Third EGRET catalog (Hartman et al. 1999) using the cluster optical redshift given in Table 1. We consider the same EGRET-cluster associations (with the 
exception of the source 3EGJ0159-3603 associated with the clusters Abell 219S and Abell 2963 because no reliable redshift is available for these clusters) which show the $F(>100 \mathrm{MeV})-$ $S_{1.4}$ correlation analyzed in the previous Sect. 4 . The $L_{\gamma}-L_{\mathrm{X}}$ correlation shown by the data (see Fig. 10) is fitted by $L_{\gamma}=$ $C L_{\mathrm{X}}^{D}$ with best fit values $C=0.06 \pm 0.01$ and $D=0.593 \pm 0.122$ ( $1 \sigma$ errors). A similar result, however, is also found for viewing periods $\mathrm{P} 1234$ which do not show, in general, the best $\mathrm{S} / \mathrm{N}$ ratios for the detected EGRET sources: in this last case we found $C=0.08 \pm 0.02$ and $D=0.328 \pm 0.120$ ( $1 \sigma$ errors). In any case, the $L_{\gamma}-L_{\mathrm{X}}$ relation shown by the data is significant at more than the $2.7 \sigma$ confidence level.

Such a $L_{\gamma}-L_{\mathrm{X}}$ correlation indicates a connection between the physical status of the cluster ICM, and of its galaxy content, and the overall gamma-ray emissivity of the cluster: such a connection is indeed expected in the viable model for the gamma-ray emission of galaxy clusters. In fact, both the diffuse emission arising from the interaction of relativistic particles with the cluster ICM and the one arising from a superposition of the gamma-ray emission associated with individual galaxies within the cluster predict a relation $L_{\gamma} \sim L_{\mathrm{X}}^{a}$ with $a \approx 0.45-0.85$. Specifically, the cluster gamma-ray luminosity produced by non-thermal electron bremsstrahlung (see, e.g., Longair 1993),

$L_{\gamma} \propto n_{\mathrm{e}, \mathrm{rel} .} n R^{3}$,

that produced by $\pi^{0} \rightarrow \gamma+\gamma$ in $p p$ collisions (Colafrancesco \& Blasi 1998),

$L_{\gamma} \propto n_{\mathrm{p}, \mathrm{rel} .} n R^{2}$,

and the one produced by $\pi^{0} \rightarrow \gamma+\gamma$ in dark matter annihilation (Colafrancesco \& Mele 2001),

$L_{\gamma} \propto n^{2} R^{3}$

naturally correlate with the cluster X-ray luminosity mainly given by thermal bremsstrahlung,

$L_{\mathrm{X}} \propto n^{2} T^{1 / 2} R^{3}$,

through their dependence on the ICM particle density, $n$. Here, the densities of relativistic electrons, $n_{\mathrm{e}, \text { rel. }}$, and relativistic protons, $n_{\mathrm{p}, \text { rel. }}$, are decoupled from the ICM density while the dark matter density is proportional to the ICM density. Note that a scaling similar to that in Eq. (3) applies also to the gamma-ray emission arising from the superposition of the cluster galaxies. Using the previous scalings and the observed X-ray luminosity - temperature relation, $L_{\mathrm{X}} \sim T^{b}$ with $b \approx 3$ (see, e.g., Arnaud $\&$ Evrard 1999; Wu et al. 1999), a correlation $L_{\gamma} \sim L_{\mathrm{X}}^{D}$, with $D \approx 0.45-0.85$ is predicted by the previous models, in agreement with our results shown in Fig. 10.

\section{Present conclusions and future perspectives}

In this paper we reported the preliminary evidence for an association of galaxy clusters with unidentified, high galactic latitude $(|b|>20 \mathrm{deg})$ gamma-ray sources in the Third EGRET catalog. Our selection criteria eventually allowed us to identify 9 EGRET sources most probably associated to 12 galaxy

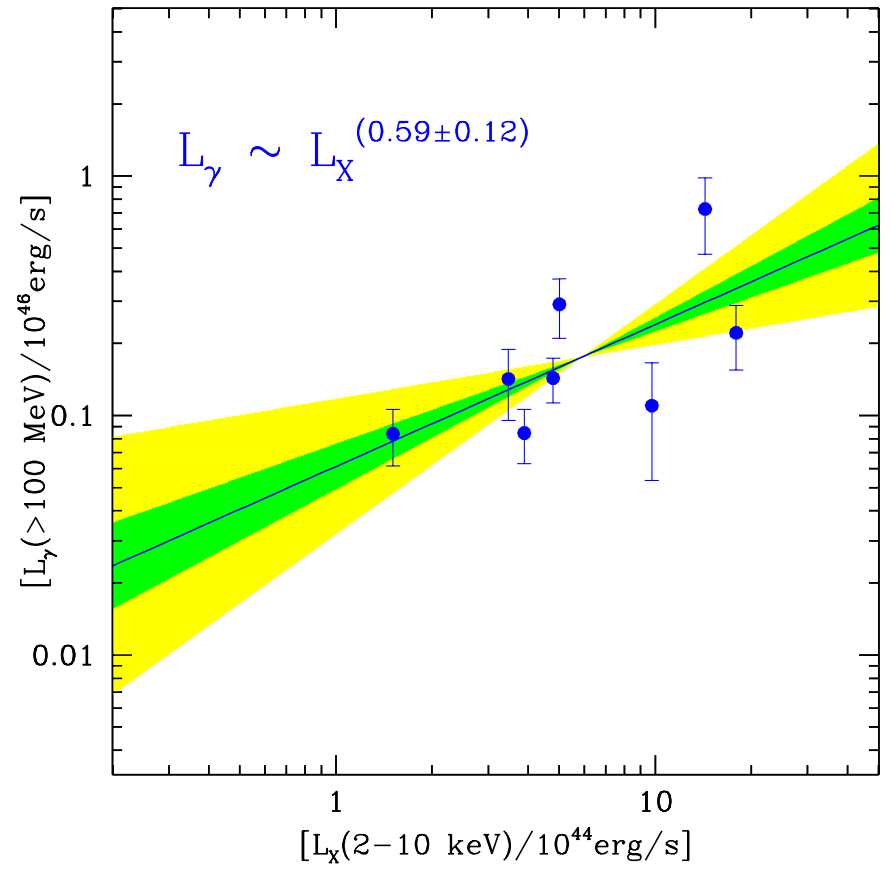

Fig. 10. The $L_{\gamma}-L_{\mathrm{X}}$ correlation shown by the clusters listed with an asterisk in Table 1. The best fit curve (solid line) is shown together with the $1 \sigma$ (green/dark-gray area) and $3 \sigma$ (yellow/pale-gray area) confidence level region for the fitting parameters. The X-ray luminosity, in units of $10^{44} \mathrm{erg} \mathrm{s}^{-1}$, is given in the $2-10 \mathrm{keV}$ energy range and the gamma-ray luminosity, at $E>100 \mathrm{MeV}$, of the associated EGRET source is given in units of $10^{46} \mathrm{erg} \mathrm{s}^{-1}$.

clusters (see the sources marked with an asterisk in Table 1) which have the following characteristics: $i$ ) the clusters are found within the $95 \%$ confidence level position error contours of the relative EGRET source map for which there is no other known counterpart; ii) the selected EGRET sources have flux $F(>100 \mathrm{MeV}) \lesssim 2 \times 10^{-7} \mathrm{~cm}^{-2} \mathrm{~s}^{-1}$ and flux variability $\$ 20 \%$ over their viewing periods; iii) the gamma-ray spectral index of the EGRET source are found in the range $\approx 2-3$; $i v$ ) the 12 galaxy clusters which are most probably associated with the previous 9 unidentified EGRET sources have bright radio sources (identified radio galaxies, radio halo/relic, NVSS bright radio source) in the cluster environment; $v$ ) the nine EGRET sources selected according to the previous criteria show a correlation $F(>100 \mathrm{MeV}) \sim S_{1.4}^{0.19 \pm 0.09}$ between their gamma-ray flux, $F(>100 \mathrm{MeV})$, and the radio flux at $1.4 \mathrm{GHz}$, $S_{1.4}$, of the brightest radio source in the associated clusters; $v i$ ) the same EGRET sources and the same clusters show also a correlation, $L_{\gamma} \propto L_{\mathrm{X}}^{0.59 \pm 0.12}$ between the gamma-ray luminosity at $E>100 \mathrm{MeV}, L_{\gamma}$, of the EGRET source and the X-ray luminosity, $L_{\mathrm{X}}$, of the associated clusters.

From our analysis of the sample listed in Table 1, we expected a priori a spatial correlation between unidentified EGRET sources and galaxy clusters at the $\sim 1.73 \sigma$ confidence level (see Sect. 2). We noticed, however, that this should be considered as a lower limit to the true statistical confidence level of the correlation since the effect of the non-uniform EGRET sky coverage has to be taken into account and it would tend to increase the statistical significance level of the 
EGRET-cluster spatial correlation (see Sect. 2 for a discussion). The detailed analysis (see Sect. 3) of each specific EGRET source yielded a most probable spatial association between 9 EGRET sources and 12 Abell clusters selected from the original list of 18 EGRET sources associated with 24 clusters given in Table 1: such a spatial correlation is found at $\sim 3 \sigma$ confidence level and might decrease to $\sim 2.5 \sigma$ eliminating the still questionable case of the spatial association between Abell 1688 and 3EGJ1310-0517 (see Fig. 4). Note, again, that this is a lower limit to the true statistical confidence level of the correlation because of the effect of the non-uniform EGRET sky coverage.

The gamma-ray-radio correlation found for the nine most probable EGRET-cluster associations,

$F(>100 \mathrm{MeV}) \sim S_{1.4}^{0.19 \pm 0.09}$,

is at $\approx 2.05 \sigma$ confidence level (we considered here only the statistical uncertainties). The gamma-ray - X-ray correlation shown by the same EGRET-cluster associations,

$L_{\gamma} \propto L_{\mathrm{X}}^{0.59 \pm 0.12}$,

is at $\approx 4.9 \sigma$ confidence level (again, we considered only the statistical uncertainties).

We estimated the diffuse gamma-ray fluxes predicted in the available models under reasonable assumption for the energy density of relativistic particles in the ICM (see Sects. 4 and 5 above) for the galaxy clusters listed in Table 1 and we found that the total diffuse fluxes are usually a factor $2-4$ below the fluxes actually detected for the associated EGRET sources. So, to recover the gamma-ray flux of the EGRET sources we have to consider that, at least, a comparable fraction of the cluster gamma-ray flux is contributed also by the (active) radio galaxies living within the cluster. We found, consistently with such a picture, that all of the clusters which are counterparts of the unidentified EGRET sources host several bright radio galaxies in their environment. Such radio galaxies can be, or have recently passed through a phase of substantial gamma-ray emission, according to the leading unified scheme scenarios for radio galaxy evolution (see, e.g., Urry \& Padovani 1995). Thus, the EGRET data require that the gamma-ray flux associated to the relative galaxy clusters are likely due to a superposition of diffuse and concentrated gamma-ray emission.

The low flux variability of the associated EGRET sources does not indicate a strong contamination from very bright $\left[F(>100 \mathrm{MeV})>5 \times 10^{-7} \mathrm{~cm}^{-2} \mathrm{~s}^{-1}\right]$ AGN-like gamma-ray sources with strong flux variability. This is clearly shown by the comparison of the flux changes for the EGRET sources more probably associated with clusters (see Fig. 1) with the flux changes of the EGRET sources spatially correlated with clusters and whose gamma-ray emission is dominated by bright AGNs (see Fig. 2).

The spectral indices of the most probable EGRET-cluster associations are found to be in the range $\approx 2-3.5$, values which are consistent with the expectations from model of the diffusion of relativistic particles in the ICM, and seem to be quite larger than the very flat spectral indices $(\gamma \lesssim 2)$ shown by the EGRET sources associated with pulsars. Theoretical models for cluster gamma-ray emission predict in fact slopes in the range $\gamma \sim 1.8-3.2$ (see, e.g., Fig. 11, see also Blasi 2000), going from annihilation of dark matter neutralinos to non-thermal electron bremsstrahlung. Active galaxies with a substantial gamma-ray emission at the flux level shown by the EGRET-cluster associations also have spectral slopes $\gamma \sim 2-2.8$, as shown in Fig. 3 (see also Hartman et al. 1999). Thus, the superposition of gamma-ray emission of both diffuse origin and coming from the active galaxies shall certainly show overall spectral indices which are consistent with those of the nine EGRET sources selected in our analysis.

In conclusion, we found that there are several converging evidence (even though still preliminary) of an association between unidentified EGRET sources at high galactic latitude $(|b|>20 \mathrm{deg})$ and galaxy clusters which show an enhanced radio activity in their ICM as triggered by radio (or active) galaxies or by non-thermal phenomena giving rise also to radio halos and relics (see, e.g., Colafrancesco 2001a,b). These evidence are found at several levels, from the geometrical spatial association with a minimal statistical confidence level of $\sim 2.5 \sigma$ (see Sect. 2), to the gamma-ray flux and luminosity correlations with the radio and X-ray data of the associated clusters with a statistical confidence level of $\sim 2.1 \sigma$ and $\sim 4.9 \sigma$, respectively (see Sects. 4 and 5).

Even though the cluster sample we derived here is far from being an a priori flux limited sample, the correlation we found with unidentified EGRET gamma-ray sources can be considered as the first evidence of the expected distribution of the gamma-ray luminosity of "active" galaxy clusters.

There have been recently other attempts to investigate the possible association of galaxy clusters with EGRET gammaray sources. In fact, Totani \& Kitayama (2000, hereafter TK) proposed that only galaxy clusters which are just dynamically forming might be bright sources of gamma-rays due to Inverse Compton Scattering (ICS) of CMB photons by highenergy electrons accelerated at the shock waves induced by gravity during the early formation of large scale structures. Their model would predict, for instance, a gamma-ray flux of $F(>100 \mathrm{MeV}) \sim 6.5 \times 10^{-7} \mathrm{~cm}^{-2} \mathrm{~s}^{-1}$ for a Coma-like cluster undergoing a merger event (roughly a factor 16 higher than the actual upper limit, $F(>100 \mathrm{MeV}) \sim 4 \times 10^{-8} \mathrm{~cm}^{-2} \mathrm{~s}^{-1}$ found for Coma in the EGRET database (see, e.g., Sreekumar et al. 1996)). As a consequence, TK predicted that a few tens of clusters $(\sim 20$ to 50 with a limiting flux $F(>100 \mathrm{MeV}) \sim$ $10^{-7} \mathrm{~cm}^{-2} \mathrm{~s}^{-1}$ ) should have already been detected by EGRET (see their Fig. 1). The absence of any correlation between the ROSAT Bright Cluster Sample (Ebeling et al. 1998) or the ACO (Abell et al. 1989) cluster catalog and the EGRET source catalog should depend, according to TK, on the large extension of these "just forming clusters" which would cause a huge dimming of their X-ray surface brightness as well as of their surface number density of galaxies in the optical with respect to the population of virialized, relaxed clusters.

However, more recently and after the submission of our paper, the same authors (Kawasaki \& Totani 2002, hereafter KT) found instead a strong correlation between merging clusters and steady unidentified EGRET sources at high galactic latitude $(|b|>45 \mathrm{deg})$. They used the same data sets (Third EGRET catalog and ACO cluster catalog) and found that 


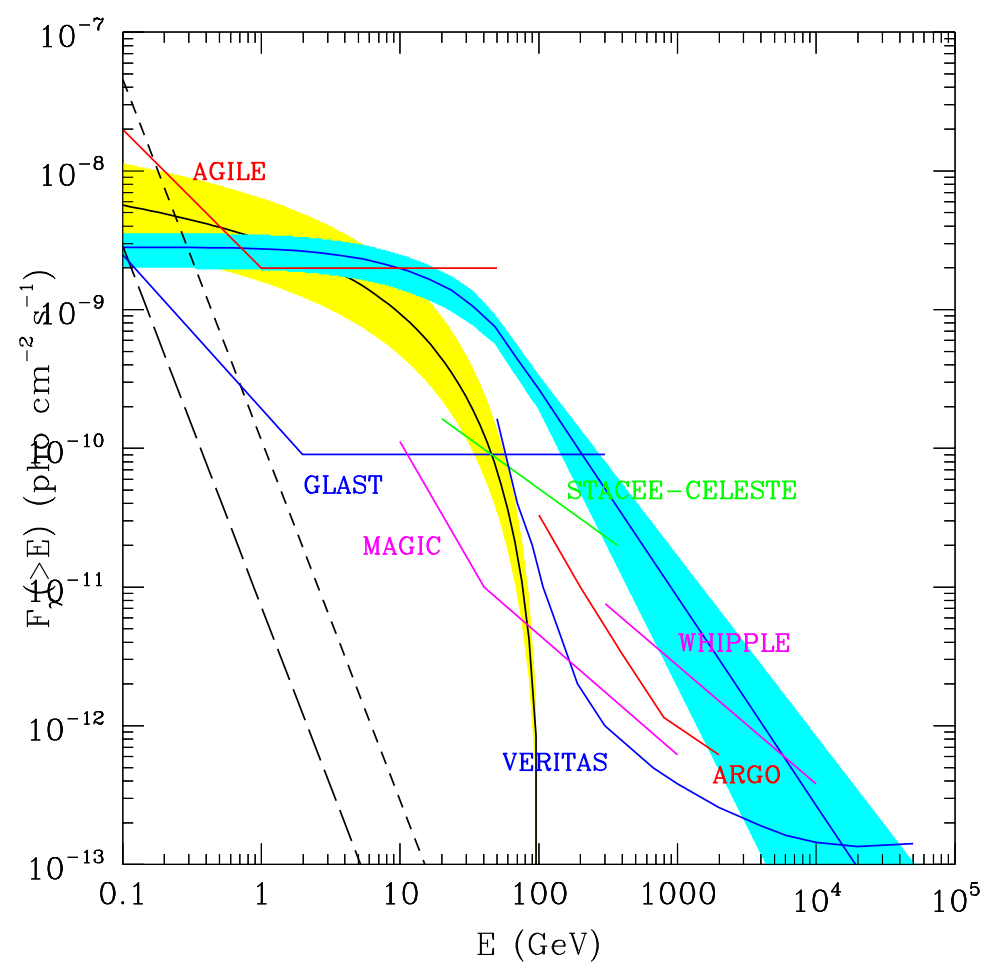

Fig. 11. Theoretical predictions for the gamma-ray flux $F_{\gamma}(>100 \mathrm{MeV})$ expected for a Coma-like cluster are shown as a function of the gammaray energy and are compared with the sensitivity of the next generation space-borne and ground-based gamma-ray experiments: non-thermal electron bremsstrahlung (Sreekumar et al. 1996; Colafrancesco 2001b) for two choices of the intracluster magnetic field ( $B=0.3 \mu \mathrm{G}$ : shortdashed curve and $B=1 \mu \mathrm{G}$ : long-dashed curve); decay of neutral pions produced in pp collisions (Colafrancesco \& Blasi 1998) (blue curve and the associated theoretical uncertainties given in the cyan region); decay of neutral pions produced in the annihilation of dark matter neutralinos (Colafrancesco \& Mele 2001) (black solid curve and the associated theoretical uncertainties given in the yellow region). Due to the very different spatial resolution of the various experiments reported, we show here the case of their sensitivity for point-like sources.

9 close pair/groups (CPG) of Abell clusters have a significative statistical level of spatial association with 7 steady unidentified EGRET sources. Interestingly, 6 out of these 7 EGRET sources are coincident with the EGRET-cluster associations found in our analysis (see Table 1) while the last case (the clusters Abell 1564 and Abell 1581 associated with 3EGJ1235+0233) is not found in our analysis because these clusters are found outside the $95 \%$ confidence level position error contours of the relative EGRET source (see Sect. 2). These last authors, nonetheless, suggested that the gamma-ray emission comes only from just forming/merging clusters with large, violent shocks, but not from usual ones in dynamically quiet regime where the violent shock has subsided. They further concluded that their finding "implies that the bulk of the steady unidentified EGRET sources in the high latitude originate from forming clusters" and "indirectly give support to the gamma-ray cluster hypothesis" delineated in TK.

Let us briefly comment on this point. We notice here that since the gamma-ray clusters considered in TK and KT are physically the same "forming/merging clusters" (their gammaray fluxes are evaluated according to the same ICS model) and since TK predicted that a few tens of these clusters should have already been detected by EGRET, there seem to be a missing gamma-ray cluster problem in their approach because KT do not find the remaining $(\sim 13-43)$ bright gamma-ray clusters, as predicted by TK. A possible solution to this problem could be that the large majority of the forming clusters are not "just forming" as suggested by TK but have experienced a strong merging event more than a few Gyrs ago, so that the gammaray emission from the once accelerated primary electrons has faded away due to their rapid energy losses $\left(t_{\text {cool }} \gtrsim 2 \times 10^{6} \mathrm{yr}\right.$; see, e.g., Blasi 2000 and TK). The only gamma-ray clusters still remaining should be those which experienced a strong merger event in the last $\sim 10^{8} \mathrm{yr}$.

But there are also other concerns as regards the energetics of the just forming/merging galaxy clusters. The gamma-ray luminosity of the EGRET sources selected by KT are found in the range $L_{\gamma} \sim 10^{45}-10^{46} \mathrm{erg} \mathrm{s}^{-1}$ (see Fig. 10: note that most of the EGRET sources selected by KT are the same we select in this paper) and should be emitted from primary electrons on a time scale $t_{\text {cool }} \sim 2 \times 10^{6} \mathrm{yr}$. This gamma-ray power should be compared with the total power, $L_{\text {merg }} \sim E_{\text {merg }} / t_{\text {merg }}$, provided by the merging between two sub-cluster units with masses $M_{1}$ and $M_{2}$, respectively. Here the total energy of the merger is $E_{\text {merg }} \approx G M_{1} M_{2} / d$ where $d$ is the typical subcluster separation at which most of the energy is released on the time scale for the merging, $t_{\mathrm{merg}} \approx 10^{9} \mathrm{yr}$, of the order of the crossing time for the considered cluster. Simulations show that equal-mass mergers are more effective in releasing energy from its gravitational form to heating of the ICM and to particle 
acceleration at the ICM shocks. Thus, the total power provided by the merger can be written as

$L_{\text {merg }} \approx 1.6 \times 10^{45} \operatorname{erg~s}^{-1}\left(\frac{M}{10^{14} M_{\odot}}\right)^{2}\left(\frac{d}{1.5 \mathrm{Mpc}}\right)^{-1}$,

(see also Blasi 2000). It is reasonable to consider that only a fraction $\varepsilon \sim 10^{-2}$ of the total $E_{\text {merg }}$ is transformed in particles which are shock-accelerated up to energies $E \gtrsim \mathrm{GeV}$ while the bulk of the total merging energy goes mainly into heating of the cluster ICM (see, e.g., Blasi 2000). Thus, the gamma-ray luminosity emitted by primary electrons accelerated at the merging shocks can be written, in general, as $L_{\gamma} \approx \varepsilon L_{\mathrm{merg}}$. The values of $L_{\gamma}$ of the EGRET sources selected by KT require, on average, an efficiency $\varepsilon \sim 1-10$ in the CGP clusters. This result seems to be strongly in contrast with the available models for gammaray emission from a population of primary electrons for which $\varepsilon \sim 10^{-2}-10^{-1}$ is expected (Blasi 2000; Colafrancesco \& Blasi 1998; Sarazin 2002).

Such a problem could be partially reduced if a substantial fraction of the gamma-ray emission from clusters is provided by $\pi^{0} \rightarrow \gamma+\gamma$ decay produced by pp interactions in the cluster ICM, where high-energy protons are accelerated at the same merging shocks but do not appreciably loose their energy over an age comparable with $H_{0}^{-1}$ (see, e.g., Colafrancesco \& Blasi 1998). If a ratio $\mathrm{p} / \mathrm{e}^{-} \sim 10-100$ is assumed, then a large part of the cluster gamma-ray emission could be dominated by $\pi^{0}$ decay and secondary electron emission by bremsstrahlung and ICS. This fact would weaken the constraint $\varepsilon \sim 1-10$ for the values $L_{\gamma}$ of the EGRET sources produced by the primary electrons in the approach of KT but would also provide a gammaray emission which is stationary with time along the cluster lifetime (Blasi 2000). As a consequence, a large fraction of the 20-50 gamma-ray, merging clusters predicted by TK should have been already detected by EGRET, which does not seem to be the case.

On another side, if the gamma-ray luminosity of the EGRET source, $L_{\gamma} \sim \varepsilon L_{\text {merg }} \approx 10^{45} \mathrm{erg} \mathrm{s}^{-1}$ is provided by primary electrons accelerated at the merging shock, then a much larger energy amount, $E_{\text {merg }} \sim E_{\gamma} / \varepsilon$, where $E_{\gamma} \approx L_{\gamma} \cdot t_{\text {cool }} \sim$ $6 \times 10^{60-61}$ erg (we assume here $t_{\text {cool }} \approx 2 \times 10^{6} \mathrm{yr}$ and $L_{\gamma} \approx 10^{45-46} \mathrm{erg} \mathrm{s}^{-1}$, see Fig. 10) should go mainly into heating of the ICM. Note also that this estimate is a lower limit to the energy available for heating of the ICM since only electrons which produce emission at $E>100 \mathrm{MeV}$ are considered. We notice that the energy budget $E_{\mathrm{merg}} \approx E_{\gamma} / \varepsilon \sim 6 \times 10^{60-61} \mathrm{erg}$ (we assume $\varepsilon=10^{-2}$ ) is larger than the kinetic energy of the IC gas, which is of the order of

$E_{\text {kin }} \approx \frac{3}{2} N_{\mathrm{p}} n k T \sim 2.2 \times 10^{59} \operatorname{erg}\left(\frac{n}{10^{-4} \mathrm{~cm}^{-3}}\right)\left(\frac{T}{8 \mathrm{keV}}\right)$

(we assume here a sphere of IC gas with particle density $n$, temperature $T$ and total number of particles $N_{\mathrm{p}}=M / m_{\mathrm{p}}$ where $M=10^{14} M_{\odot}$ is the gas mass of the cluster and $m_{\mathrm{p}}$ is the proton mass). As a consequence, one should expect that the just forming/merging clusters suggested by KT and TK have quite high temperatures if most of the merging energy is transformed into heating of the ICM, as indicated by numerical simulation (see, e.g., Sarazin 2002 for a review). Specifically, for $n \approx 10^{-4} \mathrm{~cm}^{-3}$, an ICM density which is appropriate to nonvirialized clusters, one should expect to have $T \sim 27-270 \mathrm{keV}$, values which are by far higher than the temperatures actually observed in relaxed clusters of similar mass and also higher than those of the forming/merging clusters found in numerical simulations of structure formation (see, e.g., Roettiger et al. 1999; Ricker \& Sarazin 2001; Schindler 2002). This result indicates again that the hypothesis that the EGRET source luminosity is provided by just forming/merging clusters is somewhat extreme.

Finally, we notice here, following Blasi \& Colafrancesco (1999) and Blasi (2000), that values $L_{\gamma} \sim 10^{45} \mathrm{erg} \mathrm{s}^{-1}$ provided by primary electrons would imply a quite high diffuse radio emission in the case of usual IC magnetic field values $B \sim 1 \mu \mathrm{G}$, which would have been easily detected in these merging clusters, unless very low and unreasonable (see, e.g., Carilli \& Taylor 2002) values, $B \ll 1 \mu \mathrm{G}$, are considered. Moreover, also strong EUV and hard X-ray excesses would be present in many of the merging clusters selected by KT, which does not seem the case (see, e.g., Bowyer 2000; Lieu et al. 1999; Colafrancesco 2001a).

So, in conclusion, the suggestion that just forming/merging clusters are the counterparts of the unidentified EGRET sources at high galactic latitude seems to face several theoretical problems.

On the observational side, we noticed that none of the clusters selected by KT (and found in our analysis presented in Sect. 3 above) show evidence of strong merging. In fact, strong ICM shocks are expected in merging clusters and their features can be observed in the cluster X-ray images (see, e.g., Sarazin 2002). Shocks are irreversible changes to the IC gas in clusters and hence increase the entropy $S \propto \ln \left(T / n^{2 / 3}\right)$ in the gas. Thus, one can use X-ray observations to determine the temperature $T$ and the density $n$ of the IC gas and hence to measure the specific entropy in the gas just before and after the apparent merger shocks seen in the X-ray images. Since merger shocks produce compression, heating, pressure increase and entropy increase, the corresponding increase in all of these quantities (and in particular the entropy) can be used to check that discontinuities are really shocks and not "cold fronts" or other contact discontinuities (see, e.g., Sarazin 2002 for a discussion). Markevitch et al. (1999) applied such kind of test to the ASCA temperature maps and ROSAT images of several clusters. There are clear cases, like the Cyg-A cluster, in which a change in entropy is observed at the shock front thus confirming the presence of a merger shock. On the other hand, cold fronts with no entropy change at the discontinuity region have been observed in a number of other clusters including Abell 3667 (Vikhlinin et al. 2001), RXJ1720.1+2638 (Mazzotta et al. 2001) and possibly also Abell 754 and Abell 2163.

The cluster Abell 85 which is considered by KT as a candidate for being a strong merging system triggering the gammaray emission of the EGRET source 3EGJ0038-0949 is clearly associated, instead, with a cold front (a signature of a possible early stage of merging, see Sarazin 2002 and references therein), and not with an ongoing violent merging process.

The two clusters Abell 219S and Abell 2963 associated with 3EGJ0158-3602 have very poor information available (see 
Sect. 3.4) especially at X-ray wavelenghts, and there is no evidence of merging ongoing in these clusters.

Also the clusters Abell 1555 and Abell 1558 associated with 3EGJ1234-1318 have poor information in X-rays (see Sect. 3.7) and there is no evidence of merging ongoing in these clusters.

The clusters Abell 1564 and Abell 1581 fall beyond the $95 \%$ position error contours of the EGRET source 3EGJ1235+0233, they have poor information in X-rays and there is no evidence of merging ongoing in these clusters.

The cluster Abell 1688 associated with 3EGJ1310-0517 has no relevant information in X-rays (see Sect. 3.9) and there is no evidence of merging ongoing in this clusters.

The cluster Abell 1758 associated with 3EGJ1337+5029 is a distant, bright X-ray cluster (see Sect. 3.17) which has a temperature and metallicity structure similar to that of nearby clusters with similar richness (Rizza et al. 1998). This cluster has two main clumps with irregular, unrelaxed morphology (Rizza et al. 1998). However, the presence of either an ongoing merging or a system consisting of two orbiting cold clumps is demanded to more detailed X-ray studies with Chandra and/or XMM.

The cluster Abell 1781 associated with 3EGJ1347+2932 is a bright X-ray cluster with a high radio activity in its galaxy population (see Sect.3.15). However, there is no evidence of merging ongoing in this clusters.

To summarize, the available observations do not confirm the presence of ongoing, strong merging in the cluster sample suggested by KT as the possible counterpart of some unidentified EGRET sources.

Moreover, KT also suggested that the brightest unidentified EGRET source 3EG1835+5918 is the gamma-ray counterpart of a galaxy cluster which is still uncatalogued and should be one of the "just forming" gamma-ray clusters proposed by these authors. This X-ray cluster is well outside the error ellipse of the EGRET source and "there is no reason to suspect that they are related", according to the analysis performed by Mirabal et al. (2000): also, there is no evidence of an AGN belonging to this cluster. Other reasons that do not indicate any relation between the cluster and the EGRET source are the high gamma-ray flux, $F_{\mathrm{P} 1234}(>100 \mathrm{MeV})=(60.6 \pm 4.4) \times$ $10^{-8} \mathrm{~cm}^{-2} \mathrm{~s}^{-1}$, which is much higher than the typical flux of the EGRET-cluster associations (see Fig. 3), and the very flat spectral index, $\gamma=1.69 \pm 0.07$, which is much flatter than those of the EGRET-cluster associations (see Fig. 3). Such high gamma-ray flux and flat spectral index are more typical of an AGN or pulsar (see Fig. 3) being the possible counterpart of this bright EGRET source. These conclusions has been reached also through an independent analysis of this source by Mirabal \& Halpern (2001) and Reimer et al. (2001).

We conclude, on the basis of the available observational and theoretical evidence, that cluster formation/merging cannot be responsible for most of the gamma-ray emission observed in the clusters associated with the EGRET sources listed in Table 1. As discussed in our paper, the energy release at gamma-ray energies $E>100 \mathrm{MeV}$ of the EGRET-cluster associations is probably due to a superposition of diffuse (associated with the active ICM of the cluster) and concentrated (associated with the active galaxies living within the cluster) gamma-ray emission.

While at the moment we have the first, preliminary evidence for the first gamma-rays coming from galaxy clusters, their detailed study will have a full bloom with the next generation space-borne (AGILE, GLAST, MEGA) and groundbased (VERITAS, ARGO, MAGIC) gamma-ray instruments. The next generation gamma-ray telescopes, and especially the GLAST mission, will have the spatial and spectral capabilities to confirm the preliminary result here presented and to disentangle between the diffuse and concentrated nature of the cluster gamma-ray emission.

Gamma-ray observations of galaxy clusters in the range $\sim 0.01-10^{4} \mathrm{GeV}$ (see Fig. 11 for a prediction in the case of a Coma-like cluster) can probe directly the existence of different populations of relativistic particles (e.g., electrons, protons, dark matter particles) in the intracluster medium through their distinctive gamma-ray spectral features and will open a new window on the astrophysical studies of large scale structures in the universe. Moreover, the detection of mid-energy $(\sim 10-100 \mathrm{MeV})$ and high-energy (>100 MeV) gamma-rays from galaxy clusters will definitely disentangle the leading mechanisms for the origin of the variety of puzzling nonthermal phenomena (radio halos/relics, EUV and hard X-ray excesses) which are already observed in many galaxy clusters.

Acknowledgements. The author (S.C.) thanks the Referee for several useful suggestions which contributed to improve both the clarity and the presentation of the paper. Part of the data analysis has been performed at the ASI Science Data Center with the collaboration of P. Giommi. S.C. thanks also G. Ghisellini, M. Salvati, D. Fargion, P. Sreekumar and H. Andernach for useful discussions.

\section{References}

Abell, G. O., Corwin, H. G., \& Olowin, R. P. 1989, ApJS, 70, 1

Andernach, H., \& Tago, E. 1998, in Large Scale Structure: Tracks and Traces, ed. V. Müller et al. (World Scientific, Singapore), 147 [astro-ph/9710265]

Arnaud, M., \& Evrard, A. 1999, MNRAS, 305, 631

Bagchi, J., Pislar, V., Lima, Neto, G. B., et al. 1998, MNRAS, 296, L23

Berezinsky, V. S., Bulanov, S. V., Dogiel, V. A., Ginzburg, V. L., \& Ptuskin, V. S. 1990, Astrophysics of Cosmic Rays (Nort-Holland, Amsterdam)

Blandford, R. D. 2002, in Particles and Fields in Radio Galaxies, Proc. Oxford Radio Galaxy Workshop, ASP Conf. Ser., ed. R. A. Laing, \& K. M. Blundell (San Francisco: PASP), in press [astro-ph/0110395]

Blasi, P. 2000, Astroparticle Phys., 15, 223

Blasi, P., \& Colafrancesco, S. 1999, Astroparticle Phys., 12, 169

Bock, D. C. J., Large, M. I., \& Sadler, E. M. 1999, AJ, 117, 1578

Boehringer, H., Voges, W., Huchra, J. P., et al. 2000, ApJS, 129, 435

Bowyer, S. 2000, Proc. of the American Astronomical Society, HEAD Meet., 32, 1707

Briel, U. G., \& Henry, P. 1993, A\&A, 278, 379

Brinkman, W., Siegert, J., Reich, W., et al. 1995, A\&AS, 109, 147

Carilli, C. L., \& Taylor, G. B. 2002, ARA\&A, 40, 319

Colafrancesco, S. 2001a, in Constructing the universe with clusters of galaxies, ed. F. Durret, \& D. Gerbal 
Colafrancesco, S. 2001b, in Gamma 2001, ed. S. Ritz, N. Gehrels, \& C. R. Shrader, 427

Colafrancesco, S., \& Blasi, P. 1998, Astroparticle Phys., 9, 227

Colafranesco, S., \& Mele, B. 2001, ApJ, 562, 24

Condon, J. J., Cotton, W. D., Greisen, E. W., et al. 1998, AJ, 115, 1693

Dar, A., \& DeRujula, A. 2000, CERN-TH/2000-216, preprint [astro-ph/0007306]

Ebeling, H., Voges, W., Bohringer, H., et al. 1996, MNRAS, 281, 799

Ebeling, H., Edge, A. C., Bohringer, H., et al. 1998, MNRAS, 301, 881

Feretti, L., Perola, G. C., \& Fanti, R. 1992, A\&A, 265, 9

Fusco-Femiano, R., Dal Fiume, D., Feretti, L., et al. 1999, ApJ, 513, L21

Fusco-Femiano, R., Dal Fiume, D., De Gandi, S., et al. 2000, ApJ, 534, L7

Gehrels, N., Macomb, D. J., Bertsch, D. L., Thompson, D. J., \& Hartman, R. 2000, Nature, 404, 363

Giovannini, G., \& Feretti, L. 2000, New Astron., 5, 535

Grenier, I. A. 2001, in Gamma-Ray 2001, ed. S. Ritz, N. Gehrels, \& C. R. Shrader, AIP Conf. Proc., 587, 649

Hartman, R. C., Bertsch, D. L., Bloom, S. D., et al. 1999, ApJS, 123, 79

Henriksen, M. 2000, ApJ, 511, 666

Inoue, S., \& Sasaki, S. 2001, preprint [astro-ph/0106187]

Kaastra, J. S., Lieu, R., Mittaz, J. P. D., et al. 1999, ApJ, 519, L119

Kaiser, C. R., \& Alexander, P. 1999, MNRAS, 305, 707

Kanbach, G., Bertsch, D. L., Fichtel, C. E., et al. 1988, Space Sci. Rev., 49, 69

Kawasaki, W., \& Totani, T. 2002, ApJ, 576, 679 (KT)

Kowalski, M. P., Cruddace, R. G., Wood, K. S., \& Ulmer, M. P. 1984, ApJS, 56, 403

Komissarov, S. S., \& Gubanov, A. G. 1994, A\&A, 285, 27

Lieu, R., Ip, W.-H., Axford, W. I., \& Bonamente, M. 1999, ApJ, 510, L25

Lima Neto, G. B., Pislar, V., \& Bagchi, J. 2001, A\&A, 368, 440

Longair, M. 1993, High Energy Astrophysics (Cambridge University Press, Cambridge)
Markevitch, M., Sarazin, C. L., \& Vikhlinin, A. 1999, ApJ, 521, 526

Mazzotta, P., Markevitch, M., Vikhlinin, A., et al. 2001, ApJ, 555, 205

Miniati, F., Ryu, D., Kang, H., \& Jones, T. W. 2001, ApJ, 559, 59

Mirabal, N., Halpern, J. P., Eracleous, M., \& Becker, R. H. 2000, ApJ, 543, 697

Mirabal, N., \& Halpern, J. P. 2001, ApJ, 547, L137

Nath, B. B., \& Roychowdhury, S. 2002, preprint [astro-ph/0202201]

Owen, F. N., \& Ledlow, M. J. 1997, ApJS, 108, 41

Padovani, P., Ghisellini, G., Fabian, A. C., \& Celotti, A. 1993, MNRAS, 260, L21

Reimer, W., Brazier, K. T S., Carramiñana, A., et al. 2001, MNRAS, 324, 772

Rephaeli, Y., Gruber, W., \& Blanco, P. 1999, ApJ, 511, L21

Ricker, P. M., \& Sarazin, C. L. 2001, ApJ, 561, 621

Rizza, E., Burns, J. O., Ledlow, M. J., et al. 1998, MNRAS, 301, 328

Roettiger, K., Burns, J. O., \& Stone, J. M. 1999, ApJ, 518, 594

Sarazin, C. L. 1988, X-Ray Emission from Clusters of Galaxies (Cambridge: Cambridge Univ. Press)

Sarazin, C. L. 2002, in Merging Processes in Clusters of Galaxies, ed. L. Feretti, I. Gioia, \& G. Giovannini (Dordrecht: Kluwer), 1

Schindler, S. 2002, in Merging Processes in Clusters of Galaxies, ed. L. Feretti, I. Gioia, \& G. Giovannini (Dordrecht: Kluwer), 229

Slee, O. B., Roy, A. L., Murgia, M., Andernach, H., \& Ehle, M. 2001, AJ, 122, 1172

Sreekumar, P., Bertsch, D. L., Dingus, B. L., et al. 1996, ApJ, 464, 628

Struble, M. F., Mitchell, F., \& Rood, H. J. 1999, ApJS, 125, 35

Totani, T., \& Kitayama, T. 2000, ApJ, 545, 572 (TK)

Ulmer, M. P. 1980, ApJ, 235, 351

Ulrich, M.-H., Maraschi, L., \& Urry, M. 1997, ARA\&A, 35, 445

Urry, M. C., \& Padovani, P. 1995, PASP, 107, 803

Vikhlinin, A., Markevitch, M., \& Murray, S. 2001, ApJ, 551, 160

Völk, H., \& Atoyan, A. 1999, Astroparticle Physics, 11, 73

White, R. L., \& Becker, R. H. 1992, ApJS, 79, 331

Wu, X. P., Xue, Y. J., \& Fang, L. Z. 1999, ApJ, 524, 22

Yamada, M., \& Fujita, Y. 2001, ApJ, 553, L145 